\title{
Ultraconserved non-coding DNA within insect phyla
}

Thomas Brody ${ }^{\mathbf{1}^{*}}$ Amar Yavatkar ${ }^{2}$, Alexander Kuzin ${ }^{1}$ and Ward F. Odenwald ${ }^{\mathbf{1}^{*}}$

${ }^{1}$ Neural Cell-Fate Determinants Section,

${ }^{2}$ Division of Intramural Research, Information Technology Program,

NINDS, NIH, Bethesda, Maryland, USA

Running title: Ultraconserved sequences in insect enhancers

${ }^{1}$ Corresponding authors:

Tel: $\quad 301-496-1657$

FAX: $\quad 301-496-1339$

E-mail: brodyt@,ninds.nih.gov, odenwaldw@ninds.nih.gov 


\begin{abstract}
Presence of ultra-conserved sequence elements in vertebrate enhancers suggest that transcription factor regulatory interactions are shared across phylogenetically diverse species. To date evidence for similarly conserved elements among evolutionarily distant insects such as flies, mosquitos, ants and bees, has been elusive. This study has taken advantage of the availability of the assembled genomic sequence of these insects to explore the presence of ultraconserved sequence elements in these phylogenetic groups. To investigate the integrity of fly regulatory sequences across $\sim 100$ million years of evolutionary divergence from the fruitfly Drosophila melanogaster, we compared Drosophila non-coding sequences to those of Ceratitis capitata, the Mediterranean fruit fly and Musca domestica, the domestic housefly. Using various alignment techniques, Blastn, Clustal, Blat, EvoPrinter and Needle, we show that many of the conserved sequence blocks (CSBs) that constitute Drosophila cis-regulatory DNA, recognized by EvoPrinter alignment protocols, are also conserved in Ceratitis and Musca. We term the sequence elements shared among these species ultraconserved CSBs (uCSBs). The position of the uCSBs with respect to flanking genes is also conserved. The results suggest that CSBs represent the point of interaction of multiple trans-regulators whose functions and interactions are conserved across divergent genera. Blastn alignments also detect putative cis-regulatory sequences shared among evolutionarily distant mosquitos Anopheles gambiae and Culex pipiens and Aedes aegypti. We have also identified conserved sequences shared among bee species. Side by side comparison of bee and ant EvoPrints identify uCSBs shared between the two taxa, as well as more poorly conserved CSBs in either one or the other taxon but not in both. Analysis of uCSBs in dipterans, mosquitos and bees will lead to a greater understanding of their evolutionary origin and the function of their conserved sequences.
\end{abstract}




\section{Introduction}

Phylogenetic footprinting of Drosophila genomic DNA has revealed that cis-regulatory enhancers can be distinguished from other essential gene regions based on their characteristic pattern of conserved sequences (Kuzin et al. 2009; Kuzin et al. 2012) (Odenwald et al. 2005;Pennacchio et al. 2006; Brody et al. 2007; Loots and Ovcharenko, 2007; Hardison, 2000, Bergman et al. 2002). These studies have shown that most enhancers are made up of clusters of conserved sequences that often are comprised of 5 to 30 or more conserved sequence blocks (CSBs). On average, Drosophila enhancers span $\sim 1 \mathrm{~kb}$ and are flanked by non-conserved DNA of variable length.

Cross-species alignments have also identified conserved non-coding sequence elements associated with vertebrate developmental genes (Thomas et al. 2003; Bejerano et al. 2004), and sequences that are conserved among ancient and modern vertebrates (e. g., the sea lamprey and mammals). These elements conserved between disparate phyla are considered to be 'ultraconserved elements' (McEwen, et al. 2009; Irvine, et al. 2002). Many of these sequences act as cis-regulators of transcription (Pennacchio et al. 2006; Visel et al. 2009; McEwen et al. 2009; Visel et al. 2013; Dickel, 2018). Evidence from truncation studies indicates that, in the case of a mammalian Sonic Hedgehog enhancer, the ultraconserved element is not simply a clustering of transcription factor (TF) binding sites but has a structural component that is key to its activity (Lettice et al. 2014), suggesting that such highly conserved sequence blocks fit an enhanceosome model in which multiple adjacent and overlapping transcription factor docking sites act cooperatively to regulate gene expression (Panne, 2008). Previous studies have identified ultra-conserved elements in dipterans [Drosophila species and sepsids and mosquitos (Glazov et al. 2005; Hare et al. 2009, Sieglaff et al. 2009, Suryamohan et al, 2016)]. Comparison of consensus transcription factor binding sites, in the spider Cupiennius salei and the beetle Tribolium castaneum, have been shown to be functional in transgenic Drosophila (Ayyar et al. 2010).

Adjacent CSBs within Drosophila enhancers exhibit evolutionary conserved spacing. For example, characterization of 19 consecutive Drosophila enhancers spanning $\sim 30 \mathrm{~Kb}$ between the $v v l$ and Prat 2 genes revealed, in many instances, an evolutionarily constrained substructure between sets of enhancer CSBs (Kundu et al. 2013). Linked associations of adjacent CSBs could also be due to fixed spatial requirements for 
interactions of different transcriptional regulators (see for example Gao, et al. 2008, Panne, 2008).

In this study, we describe sequence conservation between the medfly Ceratitis capitata, the house fly Musca domestica genomic sequences and Drosophila genomic sequences. The house fly and Medfly have each diverged from Drosophila for $\sim 100$ and $\sim 120$ My respectively (Beverley and Wilson, 1984). Our analysis reveals that, in many cases, CSBs that are highly conserved in Drosophila are also conserved in Ceratitis and Musca. Similar to ultraconserved sequences in vertebrates, we consider these cross-phyla conserved sequences to be uCSBs. Additionally, the linear order of these uCSBs with respect to flanking structural genes is also maintained. However, subset of the uCSBs exhibits inverted orientation relative to the Drosophila sequence, suggesting that while enhancer location is conserved, their orientation relative to flanking genes is not.

For detection of conserved sequences in mosquitos, we have adapted EvoPrinter algorithms, to include 22 species of Anopheles plus Culex pipens and Aedes aegypti. Use of Anopheles species allows for the resolution of CSB clusters that resemble those of Drosophila. Comparison of Anopheles with Culex and Aedes, separated by $\sim 150$ million years of evolutionary divergence (Krzywinski et al. 2006), reveals uCSBs shared among these taxa. Although mosquitoes are considered to be Dipterans, uCSBs were conserved between mosquito species but not with flies.

In addition, we have developed EvoPrinter tools for sequence analysis of seven bee and thirteen ant species. Both ants and bees belong to the Hymenoptera order and have been separated by $\sim 170$ million years (Peters et al. 2017). Within the bees, Megachile and Dufourea are sufficiently removed from Apis and Bombus ( 100 My; Peters et al. 2017, Elsik et al. 2016) that only portions of CSBs are shared between species; these can be considered to be ultraconserved sequences. uCSBs are found that are shared between ant and bee species, and these are positionally conserved with respect to their associated structural genes. Finally, we discovered ant specific and bee specific CSB clusters that are not shared between the two taxa but are interspersed between shared uCSBs.

\section{Methods}

Sequence curation and alignment: Drosophila melanogaster (Dm), Apis mellifera (Am) and Anopheles gambiae (Ag), the fly, bee and mosquito genomic sequences, were curated 
from the UCSC genome browser. BLASTn (Altschul et al. 1990) was used to identify non-coding sequences within other species not represented in the UCSC genome browser. Where possible, BLAT (Kent, 2002) and BLASTn were used in comparing the order and orientation of ultra-conserved sequences in reference species with dipteran, bee and mosquito test species. BLAT was not available for the Culex comparison to Aedes, but we found that the 'align two sequences' algorithm of BLAST, using the 'Somewhat similar sequences (BLASTn)' setting, was comparable to BLAT in sensitivity to sequence homology and was useful in this comparison. Similarly, the pairwise sequence alignment program Needle, which uses the Needleman-Wunsch algorithm (Needleman et al. 1970), aligned shorter regions of near identity that could not be seen by other methods.

Mosquito EvoPrinter: An EvoPrint provides a single uninterrupted view, with near basepair resolution of conserved sequences as they appear in a species of interest. A prior paper describes protocols for genome indexing, enhanced BLAT alignments and scoring of EvoPrint alignments. Readouts are comparable to those already described (Yavatkar et al. 2008).

To compare 24 Anopheles, Aedes and Culex genomes, sequences were obtained from VectorBase (https://www.vectorbase.org/genomes). The mosquito EvoPrinter consists of 20 species, including 7 species of the Gambiae subgroup and related species $A$. christy $i$ and $A$. epiroticus, 5 species of the Neocellia and Myzomyia series (including $A$. stephensi, A. maculates, A. calcifacies, A. funestus and A. minimus), 2 species of the Neomyzomyia series (Anopheles darius and Anopheles farauti), 2 species of subgenus Anopheles (A. sinensus and A. atroparvus), Nyssoryhynchus and other American species, (A. albimanus and A. darling), and two species of the subfamily Culicinae (Aedes aegypti and Culex quinquefaciatus). Mosquito genomes are documented by Holt et al. 2002; Nene, et al. 2007; Reddy, et al. 2012, and Neafsey et al. 2014.

Hymenoptera EvoPrinter: We have also formatted seven bee species, including 6 members of the family Apidae and one member of each of the Megachilidae and Halictidae families (Table 1). In addition, we have formatted 13 ant (Formicidae) species, a diverse family of social insects, for EvoPrinter analysis (Table 1). Among these are eight representative of the subfamily Myrmicinae, three representatives of the Formicinae, two of the Ponerinae, and one Dolichoderinae. For consistency, we selected a member of the Myrmicinae as input/reference sequence, and species selection was dependent on the 
integrity and completeness of the sequence. The ant and bee EvoPrinter consist of the following species, grouped according to their phylogenetic relationships:

\section{Table 1: Ant and Bee species formatted for EvoPrint analysis}

\begin{tabular}{|c|c|}
\hline Bee species (superfamily Apoidea) & Ant species (superfamily Formicoidea) \\
\hline $\begin{array}{l}\text { Apidae - social bees } \\
\text { Apis mellifera - Western honey bee } \\
\text { Apis dorsata - Giant honey bee } \\
\text { Apis florea - Dwarf honey bee } \\
\text { Bombus terrestrialis - Buff-tailed bumblebee } \\
\text { Bombus impatiens - Common eastern bumblebee } \\
\text { Habropoda laboriosa - Southeastern blueberry bee } \\
\text { Megachilidae } \\
\text { Megachile rotundata - alfalfa leafcutter bee - } \\
\text { solitary }\end{array}$ & $\begin{array}{l}\text { Myrmicinae - } \\
\text { Atta cephalotes - Leafcutter ant } \\
\text { Acromyrmex echinatior - Panama leaf-cutting ant } \\
\text { Wasmannia auropunctata - Little fire ant } \\
\text { Pogonomyrmex barbatus - Red harvester ant } \\
\text { Monomorium pharaonis - Pharaoh ant } \\
\text { Solenopsis invicta - Red fire ant } \\
\text { Vollenhovia emeryi - Japanese ant } \\
\text { Cardiocondyla obscurior - a tramp ant } \\
\text { Formicinae } \\
\text { Lasius niger - Black garden ant } \\
\text { Ooceraea biroi - Army ant - colonial raider ant } \\
\text { Camponotus floridanus - Florida carpenter ants } \\
\text { Ponerinae } \\
\text { Harpegnathos saltator - Jerdon's jumping ant } \\
\text { Dolichoderinae } \\
\text { Linepithema humile - Argentine Ant }\end{array}$ \\
\hline
\end{tabular}

\section{Results and Discussion}

\section{Comparative analysis of dipteran non-coding DNA}

Our previous study of 19 consecutive in vivo tested Drosophila enhancers contained within a $28.9 \mathrm{~kb}$ intragenic region located between the $v v l$ and Prat 2 genes, revealed that each CSB cluster functioned independently as spatial/temporal cis-regulatory enhancer (Kundu et al. 2013). The enhancers possessed a diversity of regulatory functions, including dynamic activation of expression in defined patterns within subsets of cells in discrete regions of the embryo, larvae and/or adult.

Submission of the $29 \mathrm{~Kb}$ enhancer field to the RefSeq Genome Database of Ceratitis capitata via BLASTn revealed $17 \mathrm{uCSBs}$; all 17 regions were colinear and located between the Ceratitis orthologs of Drosophila vvl and Prat2 genes. In each case the matches between Ceratitis and Drosophila corresponded to a complete or a portion of a 
CSB identified as being highly conserved among Drosophila species (Kundu et al. 2013). Submission of the same Drosophila region to Musca domestica RefSeq Genome Database revealed 13 uCSBs that are colinearly arrayed within the Musca genome. Since the structural gene and these conserved uCSBs are currently on different contigs, the absolute orientation of the Musca sequences with respect to the Musca vll structural gene could not be determined. Nine of these Ceratitis and Musca CSBs were present in both species and corresponded to CSBs contained in several of the enhancers identified in our previous study of the Drosophila enhancer field (Kundu et al., 2013). The conservation within one of these embryonic neuroblast enhancers, vvl-41, is depicted in Fig. 1. Panel A of Fig. 1 is an EvoPrint of vvl-41 annotated to show shared CSBs with Ceratitis and Musca. Green

CSBs are shared 3 ways between the three species, red letters represent bases that are shared between Dm and Ceratitis and blue letters represent bases that are shared exclusively between Dm and Musca. Fig. 1B shows two and three-way alignments in vvl41 between the conserved CSBs in the three species. In many cases the uCSBs contained known DNA motifs for TFs. Each of the CSB elements in vvl-41 that are shared between $D m$ and Ceratitis are in the same orientation with respect to the $v v l$ structural gene. However, in Musca, the orientation of elements with respect to the structural gene is unknown since the structural gene and the CSBs are on different contigs. Supplemental fig. 1 presents three-way alignments of each of the other eight uCSBs within the $v v l$ enhancer field that are shared between Dm, Ceratitis and Musca. The uCSB of vvl-49 in Ceratitis is in reverse orientation with respect to the vvl structural gene. Many of the uCSBs in Musca are in a different orientation on the contig than in Dm, indicating microinversions. We conclude that, except for microinversions, the order and orientation of highly conserved non-coding sequences in Drosophila, Ceratitis and Musca with respect to flanking genes is the same.

Many of the non-coding regions in dipteran genomes contain uCSBs, especially in and around developmental determinants, and many of these are likely to be cis-regulatory elements such as those found in the $v v l$ enhancer field. Another example is the prevalence of uCSBs found in the non-coding sequences associated the Dm hth gene locus. A previous study identified an ultraconserved regions in hth shared between Drosophila and Anopheles (Glazov et al. 2005). We have identified additional $h$ th uCSBs shared among Dm, Ceratitis and Musca. We identified a total of 16 CSBs shared between the three species, 8 CSBs shared between Dm and Ceratitis but not Musca, and 7 CSBs shared between Dm and Musca, but not Ceratitis (fig. 2 and data not shown). Both Ceratitis and Musca contain uCSBs that were in reversed orientation with respect to the Drosophila 
orthologous regions.

EvoPrint analysis of Drosophila hth sequences immediately upstream and including the first exon, revealed a conserved sequence cluster (Fig.2) associated with the transcriptional start site. Fig.2A illustrates correspondence of the $D m$ conserved region in Ceratitis and Musca. Two of the longer CSBs were conserved in both Ceratitis and Musca, one shorter CSB was conserved only in Musca, and a second shorter CSB was conserved only in Ceratitis. Two and three-way alignments as revealed by BLASTn in a comparison of Dm, Ceratitis and Musca are shown in Fig.2B. Each of the uCSBs is in the same orientation with respect to the $h t h$ structural gene.

\section{Discovery of non-coding conserved sequence elements in mosquitoes}

EvoPrinting combinations of species using A. gambiae as a reference species and multiple species from the Neocellia and Myzomyia series and the Neomyzomyia provides a sufficient distance from $A$. gambiae to resolve CSBs. The CSB clusters resolved within the Anopheles species (data not shown) are similar to those detected using Dm as a reference sequence (Brody et al, 2008). Phylogenic analysis has revealed the Anopheles species have diverged from $\sim 48$ My to $\sim 30$ My (Kamali et al, 2014) while Aedes and Culex diversified from the Anopheles lineage in the Jurassic era ( 145-200 Mya; Krzywinski et al, 2006) or even earlier.

We sought to identify uCSBs in mosquitos by comparing Anopheles species with Aedes and Culex. We used non-coding sequences associated with the mosquito homolog of the morphogen wingless (reviewed by Nusse and Varmus, 1992) to discover associated conserved non-coding sequences. Fig. 3 illustrates a CSB cluster slightly more than 27,000 bp upstream of the $A$. gambiae wingless coding exons. CSB orientation in $A$. gambiae was reversed with respect to the ORF when compared to the orentations of both Culex and Aedes CSBs. We identified uCSBs, conserved in Culex and Aedes, coincide with CSBs revealed by EvoPrint analysis of Anopheles non-coding sequences. Supplemental fig. 2 illustrates a EvoPrinter scorecard for the non-coding winglessassociated CSB cluster described in Fig. 3. Scores for the first four species, all members of the gambiae complex, are similar to that of $A$. gambiae against itself, with subsequent scores reflecting increased divergence from A. gambiae. Culex and Aedes are distinguished from the other species by their belonging to a distinctive branch of the mosquito evolutionary tree, the Culicinae subfamily and their low scores against the $A$. 
gambiae input sequence. The mosquito EvoPrinter consists of 20 species, including 7 species of the Gambiae subgroup and related species $A$. christyi and A. epiroticus, 5 species of the Neocellia and Myzomyia series (including A. stephensi, A. maculates, $A$. calcifacies, $A$. funestus and $A$. minimus), 2 species of the Neomyzomyia series (Anopheles darius and Anopheles farauti), 2 species of subgenus Anopheles (A. sinensus and A. atroparvus), Nyssoryhynchus and other American species, (A. albimanus and A. darling), and two species of the subfamily Culicinae (Aedes aegypti and Culex quinquefaciatus). Mosquito genomes are described by Holt et al., 2002; Nene et al., 2007; Reddy et al., 2012, and Neafsey et al, 2014.

\section{Conserved sequence elements in bees and ants}

Bees and ants are members of the Hymenoptera Order, representing the Apoidea (bee) and Vespoidea (ant) super-families. Current estimates suggest that the two families have evolved separately for over 100 million years (Elsik et al. 2015: Hymenoptera Genome Database: integrating genome annotations in HymenopteraMine). To identify conserved sequences shared by bees and ants or unique to each family, we developed EvoPrinter alignment tools for seven bee and 13 ant species (Table 1). Three approaches were employed to identify/confirm conserved elements (both in coding and non-coding sequences) and their positioning within bee and ant orthologous DNAs. First, Evoprinter analysis of bee and ant genes identified conserved sequences in either bees or ants and ultra-conserved sequence elements shared by both families (figs. 4,5). Second, BLASTn alignments of the orthologous DNAs identified/confirmed CSBs that were either bee or ant specific or shared by both (data not shown). Third, side-by-side comparisons of ant and bee EvoPrints and BLASTn comparisons revealed similar positioning of orthologous CSBs relative to conserved exons (figs. 6, S2 and data not shown).

To identify conserved sequences within bee species we initially generated EvoPrints of the honey bee (Apis mellifera) genes using other Apis and Bombus species. Using EvoPrints of the Dscam2 locus resolved clusters of conserved sequences (fig. 4). Dscam2 is implicated in axon guidance in Drosophila (Millard et al. 2007) and in regulation of social immunity behavior in honeybees (reviewed by Cremer et al. 2007; Harpur et al. 2019). The EvoPrint scorecard (fig. 4A) reveals a high score (close relationship) with the homologous region in the other two Apis species. The more distant Bombus species score lower by greater than $50 \%$, and Habropoda represents a step down from the more closely related Bombus species. Megachile shows a significantly lower score reflecting its more 
distant relationship to Apis mellifera. The relaxed EvoPrint readout reveals two CSB clusters (fig. 4b). Only one sequence cluster, the lower 3' cluster, is conserved in all six test species examined, while the 5' cluster is absent present in all species except Megachile. BLAST searches confirmed that the 3' cluster was absent from Megachile, a more distant species Dufourea novaeangliae, and all ant species in the RefSeq genome database (data not shown). BLASTn alignments also revealed conservation of the 3' cluster in the bee species Dufourea novaeangliae, the wasp species Polistes canadensis and two ant species, Vollenhavia emeryi and Dinoponera quadriceps.

EvoPrinter analysis of bee and ant genes that are orthologs of the Drosophila neural development genes goosecoid (gsc) and castor (cas) revealed conserved non-coding DNA that is unique to either bees or ants or conserved in both (fig. 5). The Drosophila Gsc homeodomain transcription factor is required for proper axon wiring during embryonic CNS development and has recently been linked to social immunity behavior in honeybees (reviewed by Cremer et al. 2007; Harpur et al. 2019). The Drosophila Cas Zn-finger transcription factor has been shown to be essential for neuroblast temporal identity decisions during neural lineage development (Baumgardt et al. 2014; reviewed by Brody and Odenwald 2007). EvoPrints of the Hymenoptera orthologs identify non-coding conserved sequence clusters that contained core uCSBs shared by both ant and bee superfamilies, and these uCSBs are frequently flanked by family-specific conserved clusters (figs. 4, 5, 6 and data not shown). For example, analysis of the non-coding sequence upstream of the Wasmannia auropunctata (ant) cas first exon identifies both a conserved sequence cluster that contains ant and bee uCSBs and an ant specific conserved cluster that has no counterpart found in bees (fig. 5B and data not shown). It is likely that the ant specific cluster was deleted in bees, since BLASTn searchs of Wasmannia against the European paper wasp Polistes dominula reveals conservation of a core sequence corresponding to this cluster (data not shown).

The combined evolutionary divergence in the gsc and cas EvoPrints, accomplished by the using multiple test species, reveals that many of the amino acid codon specificity positions are conserved while wobble positions in their ORFs are not. The lack of wobble conservation indicates that the combined divergence of the test species used to generate the prints afford near base pair resolution of essential DNA.

Cross-group/side-by-side bee and ant comparison of their conserved DNA was performed using bee specific and ant specific EvoPrints and by BLASTn alignments (figs. 6, S2 and 
data not shown). Fig. 6 highlights the conservation observed among bee and ant exons and flanking sequence of the glass bottom boat $(\mathrm{gbb}, 60 \mathrm{~A})$ locus of Apis melliflera EvoPrinted with four bee test species (panel A) and the Wasmannia auropunctata gbb locus EvoPrinted with three ant species (panel B). Coding sequences are underlined red, non-coding homologous regions are underlined blue, and novel CSBs present in either ants or bees but not both are indicated by the vertical lines to the side of each EvoPrint. Similarly, EvoPrinting a single exon and flanking regions of the Apis mellifera homothorax locus with four bee species and generating an ant specific EvoPrint of the orthologous ant sequence of the Ooceraea biroi homothorax locus with ten other ant species, reveals CSBs that are conserved in both Apis and Ooceraea, as well as sequences that are restricted to one of the two Hymenopteran families (supplemental fig. 2).

\section{Summary}

Our cross-species comparisons document shared ultraconserved sequences within three separate groups of insects, e.g., flies, mosquitos and Hymenoptera. In each case, CSB clusters were shown to consist of a core of highly conserved CSBs flanked by less well conserved regions. Our previous work in Drosophila has shown that most CSB clusters function autonomously as enhancers that control flanking gene expression patterns. This pattern of conservation has been documented for mammalian enhancers and suggests a common structure for cis-regulatory sequences across evolution. In many cases, the uCSBs were flanked by CSBs that were not shared across phyla. We suggest that core uCSBs perform essential cis-regulatory function(s), while flanking conserved sequences, shared only by more closely related species, serve to provide the species specificity to enhancer function. Often these enhancers control a sub-pattern of gene expression. (Perry et al., 2010, Kuzin et al., 2012, Ross et al., 2015)

In the three species groups examined in this study, flies, mosquitos, and ants and bees each have similar clusters of conserved sequences. For example, the alignment of Apis mellifera sequences with other Apis and Bombus species, or of Anopheles gambiae with other Anopheles species resolved clusters of conserved sequences resembling in many aspects BLAT alignment of Drosophila Sophophora subgroup (including $D$.

melanogaster, D. yakuba and D. persimilis) with the Drosophila subgroup (including D. virilis, $D$ grimshawi and $D$ mojavensis). These alignments revealed regions that can be considered to be, in analogy to Drosophila, CSB clusters flanked by regions of nonconservation (termed inter-clustal regions) (Kuzin et al. 2009; Ross et al, 2015). Adding 
more distantly related species, Ceratitis and Musca for flies, Aedes and Culex for mosquitos, and Megachile and ants for Hymenoptera revealed ultraconserved CSBs, nested within the CSB clusters. Therefore, the general pattern of conservation is the same for all three taxa examined.

In most cases both nBLAST and the EvoPrinter algorithm, based on the eBLAT algorithm had similar sensitivities and gave comparable results, but we recommend that the two techniques should be used in conjunction with one another. The advantage of EvoPrinter is the presentation of an interspecies comparison as a single alignment, while the advantage of nBLAST is that it provides a sensitive detection of sequence homology in a one-on-one alignment. EMBOSSED Needle alignment gives an even more sensitive detection of shorter sequences and is of use once BLAT or EvoPrinter has been used to discover shared CSBs and/or CSB clusters.

Consecutive CSB clusters in distantly related species are often co-linear, in that the order of is maintained with respect to flanking genes. We have documented exceptions to this in both flies and mosquitos in which mini-inversions (rearrangements) occur. The fact that the orientation of CSB clusters with respect to the ORF suggests that such inversions can be tolerated, and that the orientation is irrelevant to their putative enhancer function. However, the co-linear ordering of non-coding CSB clusters suggests that the order of CSB clusters may be important for gene regulation.

The pattern of conservation of CSB clusters in the Hymenoptera suggests that new CSB clusters have their origin not by recombination with other cis-regulatory DNA but random mutational changes. The same is true for mosquitos, in which shared sequences between Culex and Aedes are often not found in Anopheles. We sought to identify ultraconserved CSBs shared among bees and mosquitos that were related to those shared by Drosophila, Ceratitis and Musca, but failed to find such sequences using conventional alignment protocols. This work provides a basis for future studies to understand unique commonalities and functional differences between taxonomic groups.

\section{Acknowledgments}

We would like to acknowledge the editorial expertise and assistance of Judy Brody, Mihaela Serpe, Rosario Vicidomini, and Saumitra Choudhury. 


\section{References}

Altschul SF, Gish W, Miller, W, Myers, EW. Lipman, DJ. 1990. Basic local alignment search tool. J Mol Biol. 215:403-410.

Ayyar S, Negre B, Simpson P, Stollewerk A. 2010. An arthropod cis-regulatory element functioning in sensory organ precursor development dates back to the Cambrian. $B M C$ Biol, 8:127.

Bejerano G, Siepel AC, Kent WJ, Haussler D. 2005. Computational screening of conserved genomic DNA in search of functional noncoding elements. Nat Methods 2: $535-545$.

Bergman CM, Pfeiffer BD, Rincon-Limas DE, Hoskins, RA, Gnirke, A, Mungall, CJ. Wang AM, Kronmiller B, Pacleb J, Park S, et al. 2002. Assessing the impact of comparative genomic sequence data on the functional annotation of the Drosophila genome. Genome Bio. 3: RESEARCH0086.

Baumgardt M, Karlsson D, Salmani BY, Bivik C, MacDonald RB, Gunnar E, Thor S. 2014. Global programmed switch in neural daughter cell proliferation mode triggered by a temporal gene cascade. Dev Cell 30(2):192-208.

Beverley, SM and Wilson, AC. 1984. Molecular evolution in Drosophila and the higher Diptera II. A time scale for fly evolution. J Mol Evol. 21:1-13.

Brody T, Rasband W, Baler K, Kuzin A, Kundu M, Odenwald WF. 2007. cis-Decoder discovers constellations of conserved DNA sequences shared among tissue-specific enhancers. Genome Biol. 8:R75.

Cremer S, Armitage SA, Schmid-Hempel P. 2007. Social immunity. Curr Biol. 17:R693702.

Dickel DE, Ypsilanti AR, Pla R, Zhu Y, Barozzi, I, Mannion BJ, Khin YS, FukudaYuzawa Y, Plajzer-Frick I, Pickle CS. et al. 2018. Ultraconserved enhancers are required for normal development. Cell 172:491-499.

Elsik CG, Tayal A, Diesh CM, Unni DR, Emery ML, Nguyen HN and Hagen DE. 2016. Hymenoptera Genome Database: integrating genome annotations in HymenopteraMine. Nucleic Acids Res. 44:D793-800.

Gao S, Takemura SY, Ting CY, Huang S, Lu Z, Luan H, Rister J, Thum AS, Yang M, Hong ST. et al. 2008. The neural substrate of spectral preference in Drosophila. Neuron, 60:328-342. 
Glazov EA, Pheasant M, McGraw EA, Bejerano G, Mattick JS. 2005. Ultraconserved elements in insect genomes: a highly conserved intronic sequence implicated in the control of homothorax mRNA splicing. Genome Res. 15:800-8.

Hardison RC. 2000. Conserved noncoding sequences are reliable guides to regulatory elements. Trends Genet. 16:369-372.

Hare, EE, Peterson, BK, Iyer, VN, Meier R, Eisen MB. 2008. Sepsid even-skipped enhancers are functionally conserved in Drosophila despite lack of sequence conservation. PLoS Genet. 4: e1000106.

Harpur BA, Guarna MM, Huxter E, Higo H, Moon KM, Hoover SE, Ibrahim A, Melathopoulos AP, Desai S, Currie RW, et al. 2019. Integrative Genomics Reveals the Genetics and Evolution of the Honey Bee's Social Immune System.

Genome Biol Evol pii:evz018.

Holt RA, Subramanian GM, Halpern A, Sutton GG, Charlab R, Nusskern DR, Wincker P, Clark AG, Ribeiro JM, Wides R. et al. 2002. The genome sequence of the malaria mosquito Anopheles gambiae. Science 298:129-49.

Irvine Q, Carr JL, Bailey, WJ, Kawasaki K, Shimizu N, Amemiya, CT, Ruddle FH. 2002. Genomic analysis of Hox clusters in the sea lamprey Petromyzon marinus. J Exp Zool. 294:47-62.

Kent WJ. 2002. BLAT--the BLAST-like alignment tool. Genome Res. 12(4): 656-64.

Kamali M, Marek PE, Peery A, Antonio-Nkondjio C, Ndo C, Tu Z, Simard F, Sharakhov IV. 2014. Multigene phylogenetics reveals temporal diversification of major African malaria vectors. PLoS One 9:e93580.

Krzywinski J, Grushko OG, Besansky NJ. 2006. Analysis of the complete mitochondrial DNA from Anopheles funestus: an improved dipteran mitochondrial genome annotation and a temporal dimension of mosquito evolution. Mol Phylogenet Evol. 39:417-423.

Kundu M, Kuzin A, Lin TY, Lee CH, Brody T, Odenwald WF. 2013. Cis-regulatory complexity within a large non-coding region in the Drosophila genome. PLoS One, 8:e60137.

Kuzin A, Kundu M, Ekatomatis A, Brody T, Odenwald WF. 2009. Conserved sequence block clustering and flanking inter-cluster flexibility delineate enhancers that regulate nerfin-1 expression during Drosophila CNS development. Gene Expr Patterns 9:65-72.

Kuzin A, Kundu M, Ross J, Koizumi K, Brody T Odenwald WF. 2012. The cis-regulatory dynamics of the Drosophila CNS determinant castor are controlled by multiple subpattern enhancers. Gene Expr Patterns 12:261-272. 
Lettice LA, Williamson I, Devenney PS, Kilanowski F, Dorin J, Hill RE. 2014. Development of five digits is controlled by a bipartite long-range cis-regulator. Development 141:1715-1725

Liu F, Posakony JW. 2012. Role of architecture in the function and specificity of two Notch-regulated transcriptional enhancer modules. PLoS Genet. 8:e1002796.

Loots G, Ovcharenko I. 2007. ECRbase: database of evolutionary conserved regions, promoters, and transcription factor binding sites in vertebrate genomes. Bioinformatics 23:122-124.

McEwen, GK, Goode, DK, Parker HJ, Woolfe A, Callaway H, Elgar G. 2009. Early evolution of conserved regulatory sequences associated with development in vertebrates. PLoS Genet. 5:e1000762.

Millard SS, Flanagan JJ, Pappu KS, Wu W, Zipursky SL. 2007. Dscam2 mediates axonal tiling in the Drosophila visual system. Nature 447:720-4.

Neafsey DE, Waterhouse RM, Abai MR, Aganezov SS, Alekseyev MA, Allen JE, Amon $\mathrm{J}$, Arcà B, Arensburger P, Artemov G. et al. 2015. Mosquito genomics. Highly evolvable malaria vectors: the genomes of 16 Anopheles mosquitoes. Science 347: 1258522.

Needleman SB, Wunsch, CD. 1970. A general method applicable to the search for similarities in the amino acid sequence of two proteins. J Mol Biol. 48: 443-453.

Nene V, Wortman JR, Lawson D, Haas B, Kodira C, Tu ZJ, Loftus B, Xi Z, Megy K, Grabherr M, Ren Q. et al. 2007. Genome sequence of Aedes aegypti, a major arbovirus vector. Science 316: 1718-1723.

Nusse R, Varmus HE. 1992. Wnt genes. Cell 69:1073-87.

Odenwald WF, Rasband W, Kuzin A, Brody T. 2005. EVOPRINTER, a multigenomic comparative tool for rapid identification of functionally important DNA. Proc Natl Acad Sci U S A. 102:14700-14705.

Panne D. 2008. The enhanceosome. Curr Opin Struct Bio. 18:236-242.

Pennacchio LA, Ahituv N, Moses AM, Prabhakar S, Nobrega MA, Shoukry M, Minovitsky S, Dubchak I, Holt A, Lewis KD et al. 2006. In vivo enhancer analysis of human conserved non-coding sequences. Nature 444:499-502.

Perry MW, Boettiger AN, Bothma JP, Levine M. (2010). Shadow enhancers foster robustness of Drosophila gastrulation. Curr Biol. 20:1562-1567. 
Peters, RS, Krogmann L, Mayer C, Donath A, Gunkel S, Meusemann K, Kozlov A, Podsiadlowski L, Petersen M, Lanfear R, et al. 2017. Evolutionary History of the Hymenoptera. Curr Biol. 27:1013-1018.

Reddy, BP, Labbe P, Corbel V. 2012. Culex genome is not just another genome for comparative genomics. Parasit Vectors 5:63.

Ross J, Kuzin A, Brody T, Odenwald WF. 2015. cis-regulatory analysis of the Drosophila $p d m$ locus reveals a diversity of neural enhancers. BMC Genomics 16:700.

Ross J, Kuzin A, Brody T, Odenwald WF. 2018. Mutational analysis of a Drosophila neuroblast enhancer governing nubbin expression during CNS development. Genesis 56:e23237.

Sieglaff DH, Dunn WA, Xie XS, Megy K, Marinotti O, James AA. 2009. Comparative dgenomics allows the discovery of cis-regulatory elements in mosquitoes. Proc Natl Acad Sci U S A. 106:3053-3058.

Suryamohan K, Hanson C, Andrews E, Sinha S, Scheel MD, Halfon MS. 2016. Redeployment of a conserved gene regulatory network during Aedes aegypti development. Dev Biol. 416:402-13.

Thomas JW, Touchman JW, Blakesley RW, Bouffard GG, Beckstrom-Sternberg SM, Margulies EH, Blanchette M, Siepel AC, Thomas PJ, McDowell JC, et al. 2003. Comparative analyses of multi-species sequences from targeted genomic regions. Nature 424:788-793.

Visel A, Prabhakar S, Akiyama JA, Shoukry M, Lewis KD, Holt A, Plajzer-Frick I, Afzal V, Rubin EM, Pennacchio LA. 2008. Ultraconservation identifies a small subset of extremely constrained developmental enhancers. Nat Genet. 40:158-160.

Visel A, Rubin EM, Pennacchio LA 2009. Genomic views of distant-acting enhancers. Nature 461:199-205.

Visel A, Taher L, Girgis H, May D, Golonzhka O, Hoch RV McKinsey GL, Pattabiraman K, Silberberg SN, Blow MJ. et al. 2013. A high-resolution enhancer atlas of the developing telencephalon. Cell 152:895-908.

Yavatkar AS, Lin Y, Ross J, Fann Y, Brody T, Odenwald WF. 2008. Rapid detection and curation of conserved DNA via enhanced-BLAT and EvoPrinterHD analysis. $B M C$ Genomics 9:106. 


\begin{abstract}
Figure 1
A

gttttatgggaattttcgatgggttatataattattagctctttgttttgctatcttaaattcttgccaca gctctaagatgtttgcaacattttcattgaattcttttaataactttttactcataaatttagttttatg aaatatactgtcttctaaatcaccaaaacggta GCAATCTGAGCCTGTTATT $t g c c t t c g a a c a t t c t$

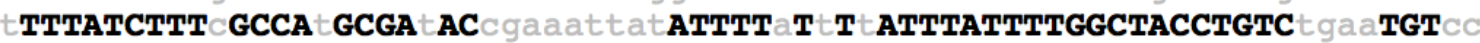
g gccat t $c c a t c g c$ TGTCTGTTGCAACTGTTGATTTTATTTCATGCCGTTTATGCAAAT $\triangle$ AAGTTCAA GGATAAGGCCAAAACAGCAGCAG $t g c c a g t t g c c a g c a t c c g a c t a c c a . g c c g$ AACATTTGGACATAAAA ATTATTTCT AATGAAATTCGT TG CCGAAATTCGTATCGATG $t c t g g c t g a a . g t c c a t c g t g t c c c t$ $t t t g c t g g c t g c c a g t t$ CAAATGCAAATGGCATGCC $g C$ CAAAA $a a$.$\rfloor AAGACCGGTAGCCGA AAAAA$ A $t$ TG $₫$ TGTGATATCGAATTAATTAAAAA gag $t c g a g c t g a g a g c c g g g c t a a j g g a a t t c c a t t c g a t$ tcgatacgatacgataccattcggat $t c g t t g c g a g a a c a g a g t$ CTGGCGCCAAATGTAAATGAGGCA $\mathbf{C}$


TTGGCGG CGTGTT $t g g g c t t t t c a g c g t t t t t c t t a a c t t t . g g t g g a a g c t g$ AGTGCGCATGCGTG TTGT G TGT $t$ TC $a g C c t c t c g t c g$ GGCGAC $T$ TGCAACAAGTTAATTAAGCCGCATTTGCATGata $t$ gtcaatggaggcagtagcgaatgctatagcagatccgaaccgatcccctgACAACCCACACTTCCTGT $g$ ga ggcacaaaaccacaaaa $\mathbf{A A A}$ ACGTGTC $\mathbf{C A G G C G G C}$ TC tagagctcggaattacccaactcctg cCgccactgccCettctactaccactcggttt tcctgggCcaaaactagcaa CGATCGATCACATGCTGC GA CTGCGGTAATCCGCACTTCCACGCCTTAGTCGCATTTTC $t$ t $t c a t t t c a t$ CGCGCCCCTTTTGT CC gacccaaa tacaggaca AGACAGGCAGTTTATTTTATGATTGATTGCAAATAAAAATCGCAAATAT

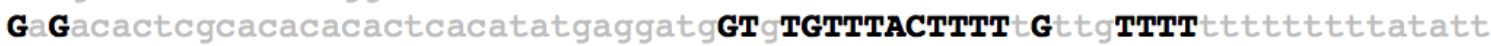
tccccgatggcatacatatagctgggcagaacccatgtggttgggttcgtgtgtgggttctggtggttcgg

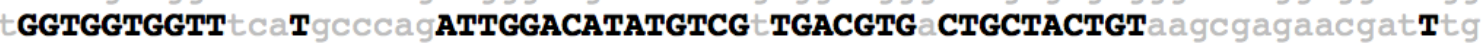

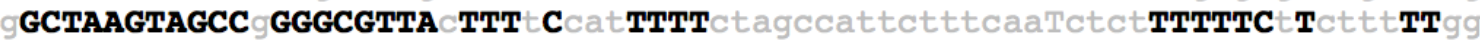
caaga TAATTATTGTCA CGCATATG $\subset$ TCA ATAT gaatgggtggtggaagggttcgatgttgtcaactttctcttatcatactaatcggttgttttgctaacta gtcattagctgaaagtcactataa.taggtaacactttctattatattatattatatactttaaagtt
\end{abstract}

B

\begin{tabular}{|c|c|c|c|}
\hline Cerat & 472349 & 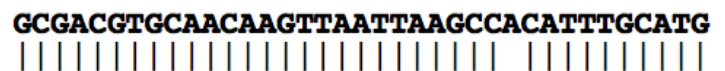 & 472313 \\
\hline Droso & 6822399 & GCEACGTECA CA GTTA TTAACCGCATTTECATG & 6822435 \\
\hline Musca & 172352 & AAGGCGTGCAACAAGTTAATTAAGGCTCATTTGCATG & 172312 \\
\hline Cerat & 472242 & $\begin{array}{l}\text { ACAACCCACACTTCC } \mathbf{4 7 2 2 2 8} \\
|||||||||||||| \mid\end{array}$ & \\
\hline Drose & 6822491 & ACAACCCACACTTCC $\quad 6822505$ & \\
\hline Cerat & 471978 & ATCGATCACATGCTGTGCAATGTGGTAATCCGCAG & ITTT 471923 \\
\hline Dresso & 6822638 & ATCGATCACATIECLISCGS & WLACECSCATIS 6822693 \\
\hline Cerat & 471502 & $\begin{array}{l}\text { TATTTTATGATTGATTGCA------AATAAAAATTGC- } \\
||||||||||||||||||\end{array}$ & AAAAAT 471467 \\
\hline Droso & 6822759 & TATTTTATGATTGATTGCA------AATAAAAATCGC & \\
\hline & 171801 & MTIMATGAAG & AAAAT 171 \\
\hline
\end{tabular}




\section{Figure 2}

\section{A}

tatttgagggccgcggagggaatttctttcaacgaggggatcccgccgatccccccgcaaaaacaaagaaa cacgcaaattgccgtcgcaaaacacaacagaatatattgcattatatattaacaatataatttgttcatc tC TAGT $a . c$ TAGTATGTAGAAGCAGCTGTGATAAATAGTGCGCATATTATACGAA $a{ }^{-}$GCAAAAA AGT

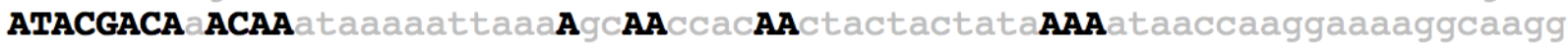
aaactcgcaaaacgagagagagaggaaaagggaaaaacgCCATGGATTTATGACAACAACAA cagtaacaatgatgttgcag CAAC tactacalacacgagtataccaagaacatc $\mathbf{A C A A C} a \mathbf{C A A}$. $g \mathbf{C}$



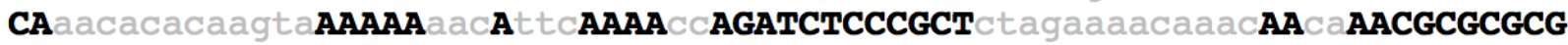

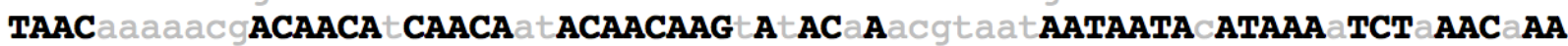
TGTGCCTGTGTTAAAA AAAA AA gC AAA $\subset$ AAGaagcaaagcgttcaaaagcaaaggettcccaata catgaaaaagtgcaaaacatatataaaatccaagaaataacatccaaaactgaagaactggaaagcc aagagtaa.tctaaggactccacacgggcagctacagtagagcttccctaaaccgaagcagttgcagcgg

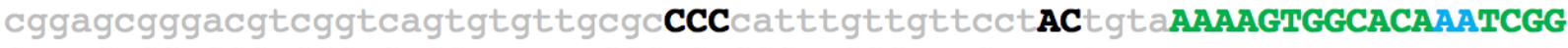
GTTAGACGTCGCTCCCAGAGCATTAATGGCTCAGCCCAGGGTAAGTAgatccaccaaagttcact tctaa atatatagaatccatatagaatggtcccccgagagtgttgttatccttaaacaagttccgacggctaaaa

B

Cerat 387653 GTTGAAGAaGCTGTGAT-ATTTGTGAGCATAT 387626

Droso 10613790 GTAGAAGCAGCTGTGATAAATAGTGCGCATAT 10613756

Droso 10613564 CATCGATTMATGA 10613577

Musca 35636 CATGGATtTATGAC 35649

Cerat 386904 TCAAAACCACATATACAAGCATTGTATACGATTTT----AAAGCAACAAAAT--TAT-TAAATTATT 386865

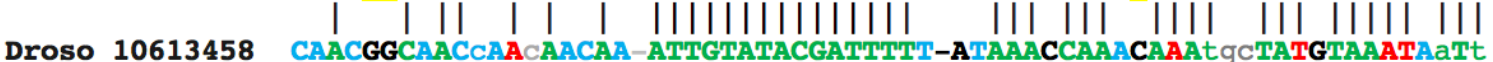

Droso 10613458 CAACGGCAACCAACAACAA-ATTGIAATACGATITIT-ATAAACCAAACAAATGCTATGLAAATAaT

Musca 35742 CAACAACAACCA-CAACAAAATTGTATACGATTTTTTCTAAAACAAAAATATAATAATTAA--AATT 35805

Cerat 386251 AAAAGTGGCACA-GGTCGGCTGGACATCGCTCCCAGAGCATTAATGGCTCAACCTAGGGTAAGTACAT 386184

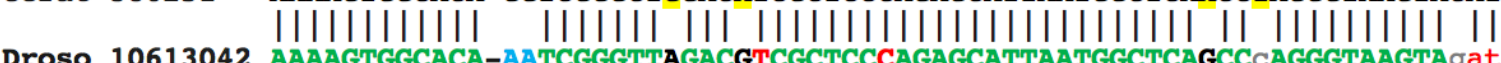
Droso 10613042 AAAAGTGGACA-AATCGGGTTAGACGTCGCTCCCAGAGCATTAATGGCTCAGCCCAGGGTAAGTAgat 10612975 Musca $383950 \quad \prod_{\text {AAAAAGTGGACAATCGGGTTGACACCGCTCCTAGAGCATTAATGGCTCAACCTAGGGTA }}\|\| \|$ 


\title{
Figure 3
}

\begin{abstract}
A
tattgaataagaaacgtaggtttaa.tcgaaattgatgatgatatttttatcaaagaagacattattg tccctttatcctcccccaaagaaacacccatcgtagcttattaagttcccttcggccccgcacttgggag t ctgccagaagagccalacggtgctagttgGAGAGTGCTGCTAATGTGTATGACTCAATTACTGTGAA AАTTMAATATTATTCGA $g c a g c a c c g c c a g t g c t g a g a g a g a . g a a$. GGT $g$ A ATAAAATCATAAAA ATACCACAC $a c c g g c a c t t g g g c c g c a c t c a t g a g c g c$ TAGAAAAGTGCAT $t c a a t g t g c a g t g a a g a g c$ atccttcgtctcggtggaatgaagagagggggtgattgtaaagaa.jcaaggggagagaTTGTTTGaGG AAAACACTTTAGAAATAAATATGA CTTCTATCG $g$ GTGACTTTTATCTCCATAAATAACGATTTATG

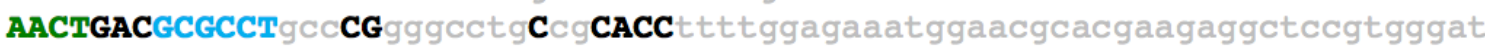

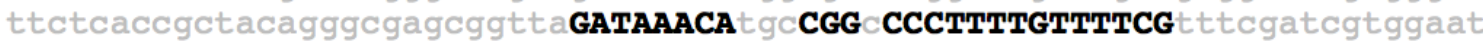
tggaaa.gc gcagcgaacgaggttggtgatgaagcGT 9 TGTTMAMAcaggcgacggaacgagccggcgtg a.GAGTGACATTCTCCG_GC gaaaatggtcgaaagaagtgcacggggcacacgatcgcgtttgagagtct ttggagtgacaggtgcatatctgttacaagatcgttgtgatcttctccatgtgctcgatttaaccggtctg ggatactgatttttttgaagatttcgatcgatccggtttgatcgtaaagtatccgtaatagtgtttaaact

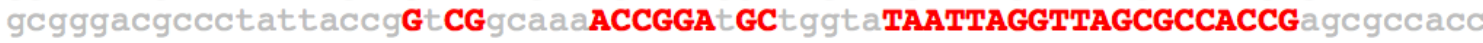
tGGCGCcacccaatagagttgaaltggcgtgaattggtataattttTGTCTtctatgtttcactccggta

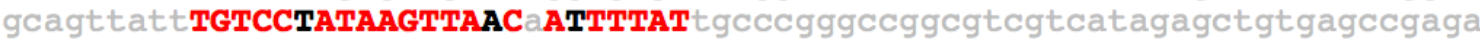
tattatgagttagatacagacggacg GCGacatttagctGGaaT $\mathbf{G} \mathbf{G T} \mathbf{T G G A G C C}$ gtgtagcaaagt ctaa.tgctgtgtctgatttttcttggtttgatgagatttcagaagaaatgacttgccctcccgccaaaaa attctccaggaacaaacacctgtttaatgttttccctgaactcaacgtttagtttaaacggaatggatag
\end{abstract}

B

\begin{tabular}{|c|c|c|c|}
\hline ulex & 610788 & CTATGTAATGTGTTATGATTCAATTACCCAGAAAAATTCAAATATTGAT & 610746 \\
\hline & & $T$ & \\
\hline pheles & $\mathbf{4 2 0 0 1 0 5 8}$ & ATTACTGTGAAATTAAATATI | | | | | | | | | | & \\
\hline
\end{tabular}

\begin{tabular}{|c|c|c|}
\hline ulex & 610664 & GAAAACACTTAAGAAATTAAATATGT--ACGTTCTATCG--CTGACTMTCATCTCCATAAAT \\
\hline eles & 42000806 & CACUITAGAATTAAATATGA--GCATTCTATCGGGCTGACTITTATCTCCA \\
\hline
\end{tabular}

\begin{tabular}{|c|c|c|c|c|}
\hline Culex & (cont'd) & AACGCTTTT-ATTAACTTTATGCATCGTCGTCCC & 610591 & \\
\hline Anopheles & (cont'd) & AACGATTTT-ATGAACTGAC--------GCGCCT & 42000731 & \\
\hline Aedes & (cont'd) & AACGATTTTTATTAACTTTACATGCAGTGCGCCT & 406893546 & \\
\hline Culex & 605426 & GTCGGACAGACAGGAAGGTGGGATAATTAGGI & $\begin{array}{l}\text { CGCCACCAAG } \\
\|\|\|\| \|\end{array}$ & 605381 \\
\hline Anopheles & 42000289 & 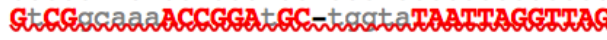 & CGCGACCSGAG & 42000245 \\
\hline Culex & 605259 & $\begin{array}{l}\text { TGTCCCATAAGTTAGCGA-TTMAT } \\
|||||||||||||||||| \mid\end{array}$ & & \\
\hline Anor & 2000157 & 2000134 & & \\
\hline
\end{tabular}


bioRxiv preprint doi: https://doi.org/10.1101/696005; this version posted July 11, 2019. The copyright holder for this preprint (which was not certified by peer review) is the author/funder, who has granted bioRxiv a license to display the preprint in perpetuity. It is made available under aCC-BY-NC-ND 4.0 International license.

\section{Figure 4}

A

\begin{tabular}{|c|c|c|}
\hline \multicolumn{3}{|c|}{ Apis_Mellifera (Bee) (Ref Sequence) } \\
\hline \multicolumn{3}{|c|}{ Composite eBLAT } \\
\hline Score & Start & End \\
\hline 2808 & 1 & 2808 \\
\hline 322 & 717 & 1780 \\
\hline 227 & 438 & 1652 \\
\hline
\end{tabular}

Bombus_Impatiens (Bee)

Composite eBLAT

Score Start End

$860 \quad 261 \quad 2462$

$196 \quad 481 \quad 1806$

$159 \quad 677 \quad 1811$

- $1^{\text {st }} \bigcirc$ All Alignments $\bigcirc$ None
Apis_dorsata (Bee)

Composite eBLAT

Score Start End

$2388 \quad 76 \quad 2788$

$284 \quad 700 \quad 1654$

$245 \quad 1223 \quad 1796$

○ $1^{\text {st }} \bigcirc$ All Alignments $\bigcirc$ None

Habropoda_Laboriosa (Bee)

Composite eBLAT

Score Start End

$\begin{array}{lll}762 \quad 287 & 2453\end{array}$

$259 \quad 681 \quad 1810$

$\begin{array}{lll}142 & 1140 & 1947\end{array}$

- $1^{\text {st }} \bigcirc$ All Alignments $\bigcirc$ None

Apis_Florea $($ Bee $)$
Composite eBLAT
Score
$2299 \quad 61 \quad 2808$
$253 \quad 1143 \quad 1826$
$195 \quad 968 \quad 1853$
\[ 1^{\text {st }} \bigcirc \text { All Alignments } \bigcirc \text { None } \]

Bombus_Terrestrialis (Bee)

Composite eBLAT

Score Start End

$917 \quad 261 \quad 2497$

$\begin{array}{lll}127 & 881 \quad 1800\end{array}$

$122 \quad 1305 \quad 1985$

- $1^{\text {st }} \bigcirc$ All Alignments $\bigcirc$ None

B

aaacgttatatatttattatatgtataatatttgaatagtgttttgtgacagtcatgacaatcattgacta ttatatatatataactcaaatcatcaagttatattgagagttcaagacaactcgttgataatatatatccaa tactatcgtagcgatatatttttcgataatcgttaataattcattgacaag.tgtgaagcaataattttta caaagttttttgaaattatctaaatcgattgatctctggaacaccctatatatcttgttgtatgttctcg

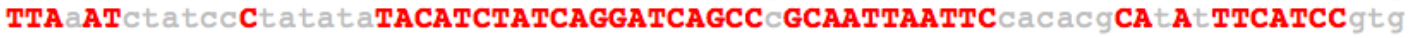
cgaatGTacacatt $\mathbf{A C A C a g g a t t a c c g c c a t g t c c a c t g t c t c g a a t t c t t c g a c a a g c a a c g a t t t t t ~}$

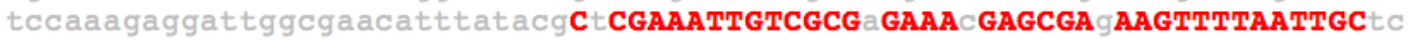

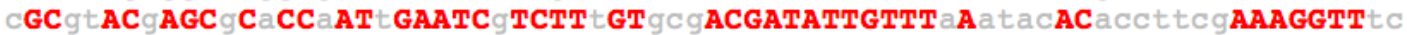

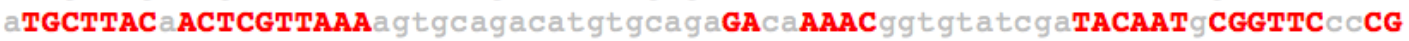
TTTCATTGTtccgaacatacaaacacattgttagatttctttcctttttttttatttttttttcatattttt

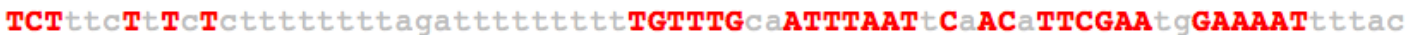

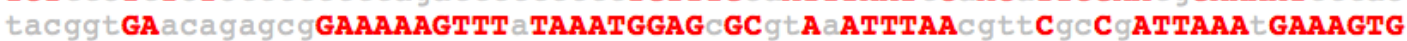

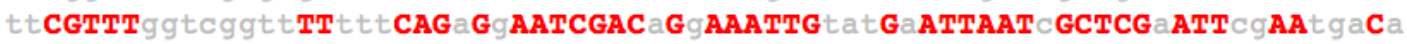
aTAATgtaaaggattcatgtaatgttttttattaaatttaatttaataaaaaaaggaaagacaagata tttcagttttgcaacagaatgaatcattcaaactgaaatagaaatattttaca.tatcgttttgttttgact atttttgtttatttctctttttttgtaatttttttcttttatttactttttttaagtcatattttaaatgt tttttgaaaatattttacgtattttttattttttatttcactataagttattaataatgatattgtaaaa ttttatataatttatcatgtaaacattagtattttaaattgcaattataacatatcaaa.tatgtaagta tataaagttttattagttttacatattaatattatgcaaattttatctatttattaagttagttttcattc atctttccaatataattgtaaaattatttatctttcttttactatttaacatcatgcaacaaataatttca aatatttcttatcattataattctaaaattatttaatgatctttttctttctaacaattttagaaagaaa ttatttatacaaatatttataactttttattcttcaaacaaataatttatgtcaacttattcaaagtat catttttctcatttattaaatatatgttttgtttaaacagttaacatttaaatattgttttttcaatatt atcaacaaattcaagatattggaataataattaaaaatattcgaaaataattaa.tagtctgtatatac atatttcaatttacttataaatatatatatacatactcttactatatatacatacttatatattatttataa atatgatctatttcgatcactattctaaaaaattaagaaaaata.aaaaaaaaggaaaacaacaatc taatttagaaatgaacaacaatctaacacaacaattgaatacaaaactcatattctttcctttcttttta ttatttaatgaaacgcatacacaatatatttacaaattctactcacgta.tcaaccttgaaccgacgcat

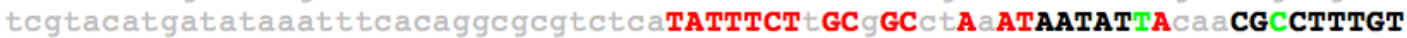

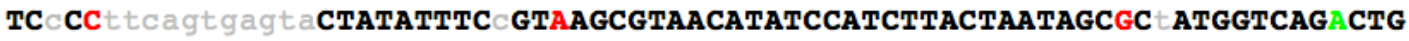
CACGTAATTGAGG $\mathbf{A G T C a c c a a c g g t t c c c c c t g t g g t a t a t a t c t c c t g t A T G T a C A t g t c g t a t a t g ~}$

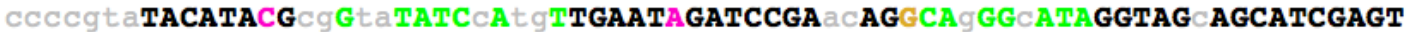
GT ATACCGAAA CTACATAACC ATCCAAGCCGGCTGACTTGGCCGACAATCGCTATTTAG $9 a . C C G C T T A$ TCTC $\mathbf{C C}$ GTCTAGCTGa gAaTcttagatagaaatgttgtgtttagaacgaagcaagaaaaaggaaaactaaaagcgagaagggaaaga ggaaaaaaattgaagggataaggaggtaaaaagaaatggcaaaggtgaagagaaagtcgtgaggggaatg ggggtgagagtggtcgtgaaaatggaagaaatttggagcaaggatgataatacgaaaagagaaaagtgagaa ataggatagagcaagaggaggagtgagtaatatcatagaaagaataagaatcaaggaaaggattaaaaag atacaatttattggagagaaaatatgaaaggattattgttaaatatgtatagttttatgtcaattttatt 
bioRxiv preprint doi: https://doi.org/10.1101/696005; this version posted July 11, 2019. The copyright holder for this preprint (which was not certified by peer review) is the author/funder, who has granted bioRxiv a license to display the preprint in perpetuity. It is made available under aCC-BY-NC-ND 4.0 International license.

\section{Figure 5}

A

tggaatatgttgaagcaacgacggaatatttcgtatataattttgattcaaaattgaaatcgattgattaatttattataa aatgaatgtctttgtcgaatgctttccttcatcttcaggaaatccttggttaattcggaaataatagcttgccccgcggt tccgcgtatattacacgcgtccetcccccccgcggcgtatggatctcgtg GCG AAAAATGAAAGAAGGCGAAATCCTCG

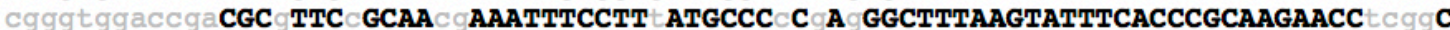
GCAGATTGGGG TGCGC $g$ CTGAAATCCGCAATCCGTTTCGATCACGC CGAACGATTTAGCT CTTGCAATCCCG $g t t$ GGAAGA $t g$ AAAACGAGAAGGAAAATATGCAAC $g$ gcatcggcg g ttctcaatggagagagagagaggagcactaggggca cggaaagagaaagagggagagagagg gagagagaga GAGAA gcgcgagtgac gcc gC GTTGAAAGCATAA $g c a t c C a$ CCC TTCGCACATTTTTATGC G GACGTCGCCTCTAAGCAGCTTTCTTC AGATTTTAAACCAAAACATCCCTTGGGaCCC $G Q$ gatggttcccagtattttttcccacttcccttttcacgcggaataaggttgcgaattgttgagaaacgttattacaatttt cgtcgctctatcggcgataaacgcgaaaccttcacgtttcgattaatcttctttaactcctccttggtcgtttccttc cttccatttttaagaaatagtaacgctttttaaagtgatctaacaggcatttgttttactcttatttgggaggctaagaa tgacgtttaatgaaaatccataagaaagaatattttttcatatctttttacgaaattctctaaatacgtaaatatat aacatacttcctttatgattctcttcctttcgtttcatcgatgtcttcgatcgaaacgaaatgagaaatgatatctgataa gaatatagttcaactattcattatttccagaa.tggaacgattattatcaaagagatggctcttttgataaaatttttcg

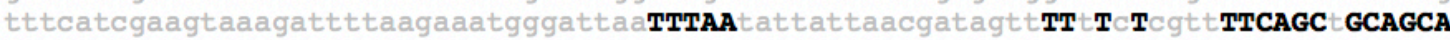
TA CC $\triangle$ TTT TA TCCGG GG TACGT GC G AATGGCAGC

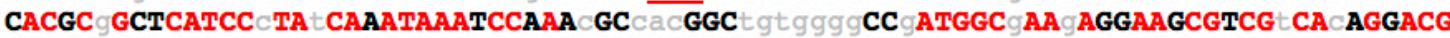
ATATT $\subset$ AC $\subset$ GA GA CA $\subseteq$ TAACAA T GAAGC AC TTCGACAAGAC CACTA CC GA GT AT


acacgtgtattgcagtacatgatta.gaaatttcagttttttgaatgtaagatttttctttttgaagaa.tattccccact agtgtttgtaatttggatttatttttgttcttcgtacttatattgatagtttttctatttttcaattaaattcgttaaat

\section{B}

tgacaccggcgcttattattaa.tcggccggagttactcgacttcgcgctggagaggcgatccgattcgcgtgagtgccga gagaaactttcgcggtttcgcgtagaataatgtgaggcaaaaggcactgtcttatccaaagatgtatatttcttcgtt gtagcgaga GGGAGAATAATacgcgccgaa TTCTCCC $t g c c a c a \mathbf{C T T T T T} c c c t c t c g g t a a c a c t c c g t t c c t c t t$

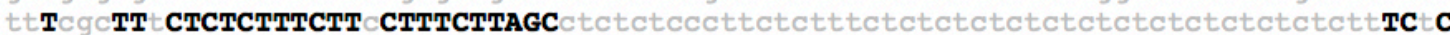

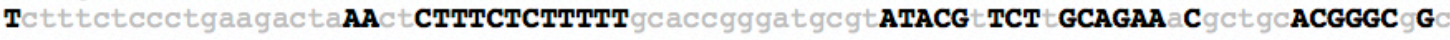
CAA GTACGC $g g g t t c c c t c \mid \mathbf{T}$ CT ACGGACATATACCAC 9 GAC GGGATCCCCG GAGCC CGAGAAATAT TCGACAATTTTTAAACTCGTTCCTGA Cac gC GGCATTCAAAC $g$ CC GCGC.$c c a g c c t c t c g c a g t c a g$ CAAGTA GAAT

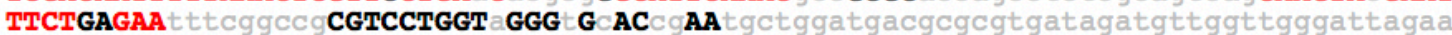
cactcgagcccgaggaggacactccgggtttcgaccgatgtgacacgtgatttgttgtcgttctgtgatcggccccgcgct gcgtccgattaatcaacgagaagcgcagtcgcgatgcgcctttgttcgtaattcgctgatatggcccgactaatacatata cgattatcctttcatgcatcaaatctctatagccactcaattgtgtagatttgcatttcttgggtgtacaatgtatcaatc tagtgcccggcgggaaagtacgagtataggacggctcgtggaagagaggtttgccgcgtccaccggtattcgacatgcccg

AATAATAAA TTTAAATAC T $\triangle$ GGCAAGAATG AAATG GCCCT GAGA GCTTTAACTGT GAAT TTCGTTT

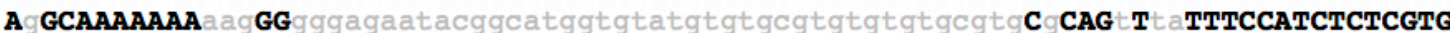
GCGCATTACCTCGGGAGATAATTATCGC AGCGAGGATTGGTCAACCGAATTGCGTCT GCGCTCAATATTTTCTTGCGT

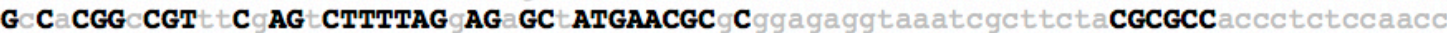

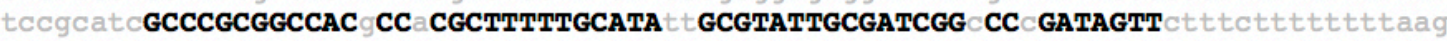

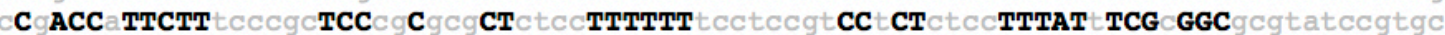

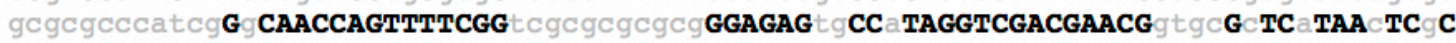
G TATGTGTATCAGag tcat $t c c g g t a t g c g c c g t t \mathbf{T C} \mathbf{T C T G} c g a t \mathbf{G T G A A C G C}$ aacccgtattatccagacc gata

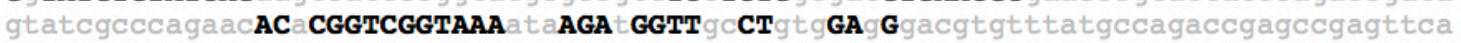
tatcgaccgaatacccatctggctcgcacggaaagagacgaagacctcgcgcagatttctagggcagtttgtgatctactg agatctgtcgagctttgaatttgaaatttgattttgatagcaacttttagacatttatagtatttcacaaatattcactt attataacacgttcattgtacattaaatcaaa.tatattataacgtaatctactttgaataagaacggagaattgttgt cgaa.tgttcttgcttatttgatttgattttttgtgtaacattttcttcttgtatattttacaaatatttcacctattata ttatacactagatcaaaattatattaacgtaatatctactttaagttagaaacggagaattgtcgttgaaatattctgct tatttaattttatttttcgtgtaatatttttttgtacgttgaattaatttaaatatgtagtcgattcaatttaatatataa

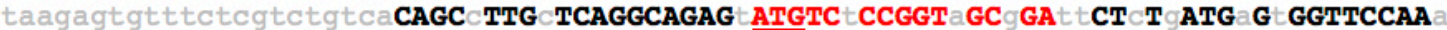

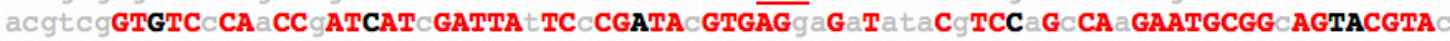

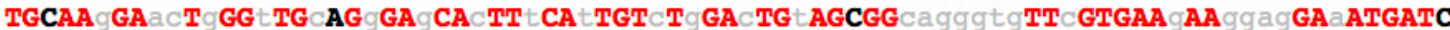
AGGCACTTCAAGTGGCA AAGAA $\triangle$ AG GACGA TC $\triangle$ TGCAGCA $\triangle$ G $\subset$ TTCATGAG TA TCGCCGA GGACGA TG TCG

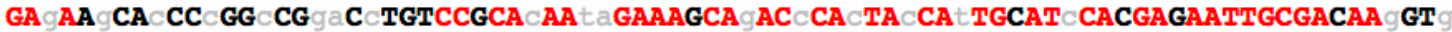
TA $\$ AT TC AC TCGGACGT CA $\bigcirc$ ATGCACGC AATTACA CG $\triangle A A$ GACTC GC $\triangle$ ATCATACA GAGGG TTCCAGCGA

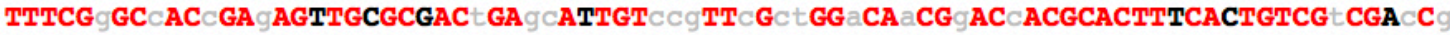
GgaTGTCGGTTCAC TTCAAAAATAA GCCGATATGGGT $g a g$ AAcgaactgtt $g C g$ TGAT $\mathbf{A}$ TG TCGC $t c t a g a t c g$ agactattcatc $\mathbf{A A T C}$ gaag $\mathbf{C C T T}$ tcactagaacttaatgtgtcgcaacgtttcggatttaattcttacgctgagagaa cgattacgttgatacatttttatttgatgtacatatctaattatattttgtcgttatttcagatctctccatgatttgctc tacaagttttcttttattttatttttcagacaagcacaagtcttatcacatcaaggacgaacaactgtcgcgcgacgg 
bioRxiv preprint doi: https://doi.org/10.1101/696005; this version posted July 11,2019 . The copyright holder for this preprint (which was not certified by peer review) is the author/funder, who has granted bioRxiv a license to display the preprint in perpetuity. It is made available under aCC-BY-NC-ND 4.0 International license.

\section{Figure 6}

Apis_Mellifera (Bee) gbb Genomic Relaxed EvoPrint

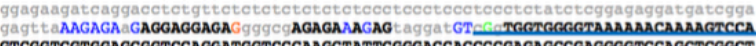

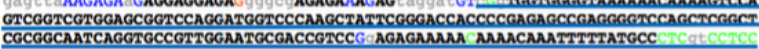

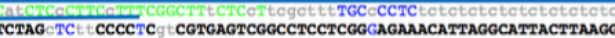

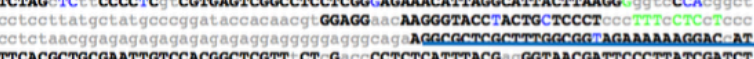

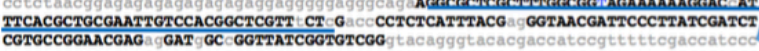



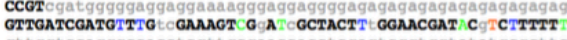

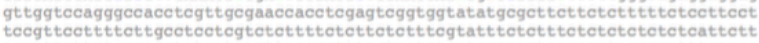

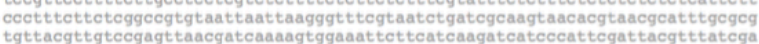

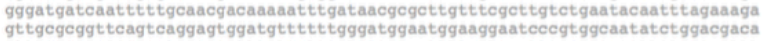

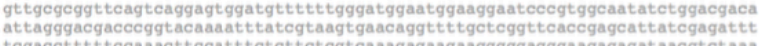

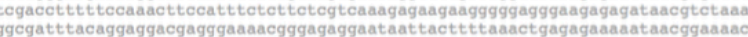

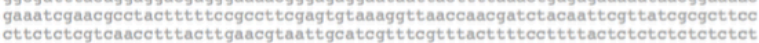
ctotctocct tgcaatcccatcacatatogogttctatctacgagataaaaatccatocacgtttgcaaggaagg aaggaaggaaggaatttgtoggaaagaattcgatccagcogagcgataaagattagaagaacgatttgccaaat

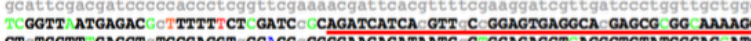



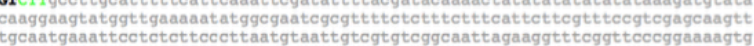

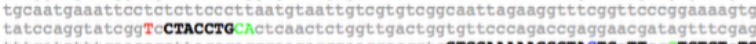

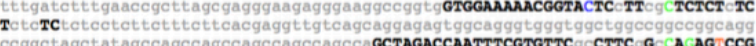

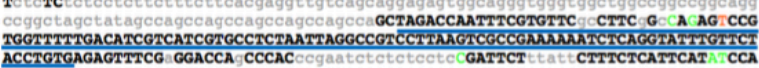

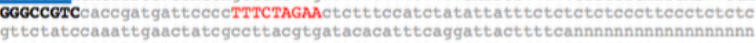

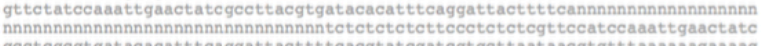
goctegogtgatacacatttcaggattacttettcacgtatogategtgottaataacgtgtt taaaaaagaaaag

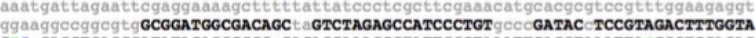

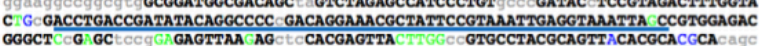

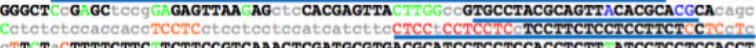

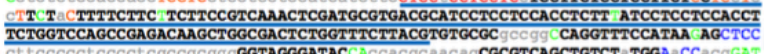

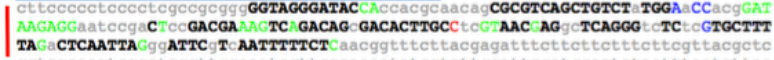

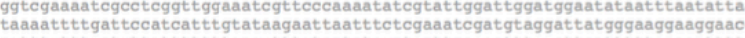



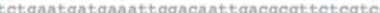

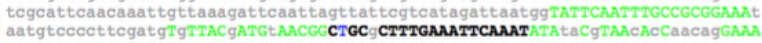

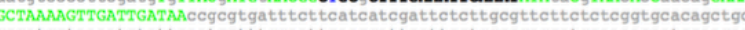

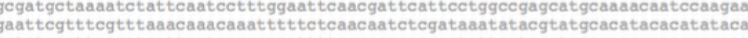
cgtatcacttaaaatcattcoctcgcaaaaattttagcaacaacgaatattactcaataaagaaagaaagaagaat

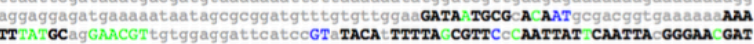

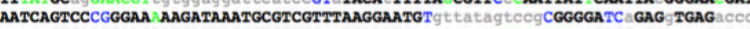

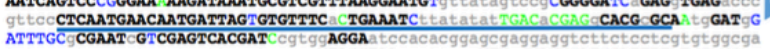

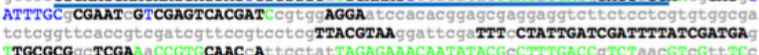

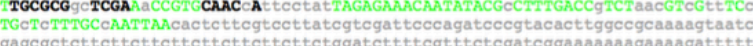
Haatagagaggataaattggttttacgaagggaagggggatgcgttgatcteaaatattegcogctgtectctetctt cegtattcacgtttcgttatcgcgatcgtttattttgttogegagaattcggtaattttcgoggacggagcgaga

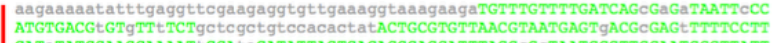

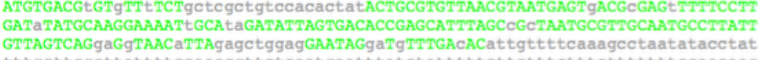

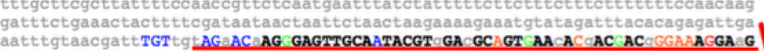

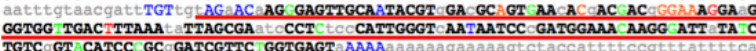



Wasmannia_Auropunctata (Ant) gbb Genomic Relaxed EvoPrint

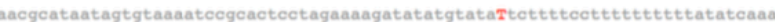

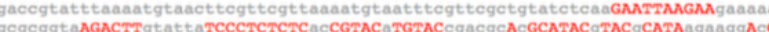
CGGAGGGGGAGGCGATCGGAMGGTACAGCGCGGCGatggtggeggoggeggeggego

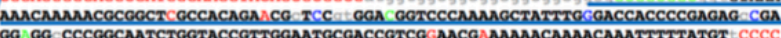

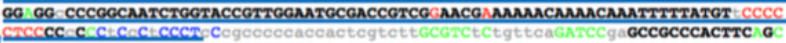

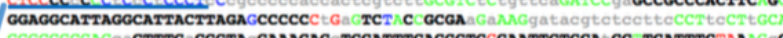

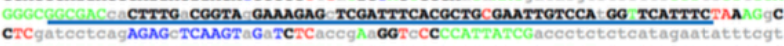

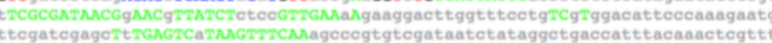
togtaaagtgggetgcattaggtagaatettceggtgaggatggggaaacaatttctcaagatgegttaccaage totgttaaacgtgcaaacctgttaaaccectgogetcttettgagattatactat tgaaaccgaatcatt ttcag atcgttatctccetctggctatcttcagcatgaacatgtactaacgttatacgattttggtatcatgcataacac acagcgegatctaataataagaaacttaaaataaaaatcacataaaataaagaaccogtcagaaaaatattgtce

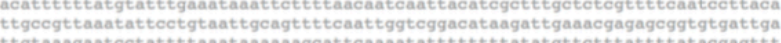

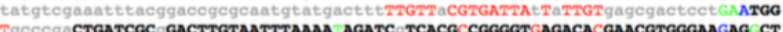

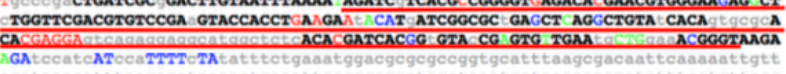

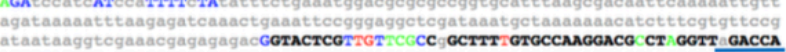

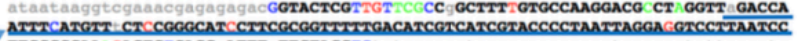

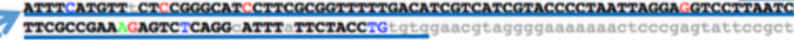

ggacaataggtgttttcagotocttttt:

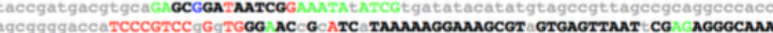
CCTC ACGCCGCCGOTM G

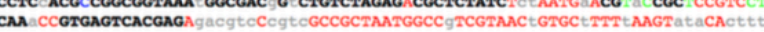

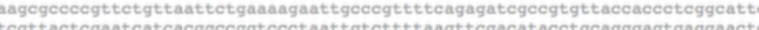
tgaccgaaaattttttagattttaatcagcgaagttgttetttcggagagagagagagttcgggegtggatggt agggtcogaggtttatccaaagtttgaatotttcattetcgeaagttteggecacctagtacggggectctttgC

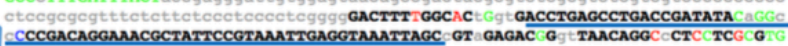
CCCGACAGGAAACGCTATTCCGTAMATTGAGGTAAATTAGC GT GAGAC G TAACAGGCC CTCC TCGCG

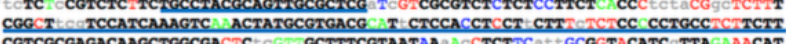

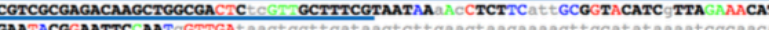


Fa .

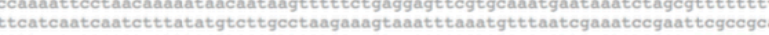

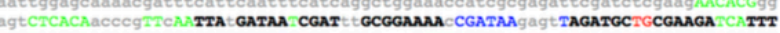

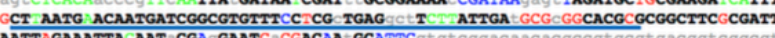

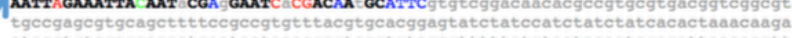
atacctgtcgcgcacaatcaataataaacgcgtccgtgtagcgtttttatgtaatacaatcgagattaaacct: ctcaaacgaactgagtttctcgaagactgaagcttaaaacacgtttgtaggatatatt tcaaacgtattttca

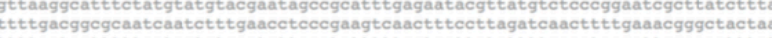

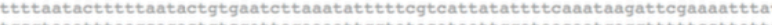
ctactgtttgttgtaaatttgactggatcataaagatacagccotctogtctcaarmCagaACtCaC GAMTAC

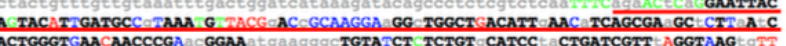
ACTCGCST 


\section{Supplemental Figure 1}

$v v l-36$

Cerat 455483

Drase 6816217

Musca 216640

vvl-37

Cerat 459528

Drase 6817479

Musca 207477

Cerat 459788

Drase 6817584

Musca 207708

$v v /-38$

Gerat 462472

Drase 6818852

Musca 202227

Serat 462706

Drose 6819085

Musca 201657

$v v l-49$

Cerrat 512064

Drose 6834923

Musca 105415

vvl-51

Serat, 517380

Drose 6837429

Musca 95350

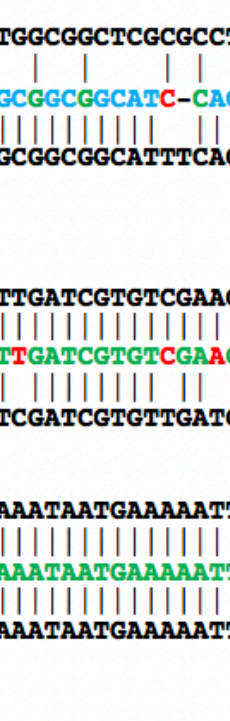

CTAAAACATTTGATGTTAATTGATTTAAACAACTTTGAACTTGTTGATCTTGCAACAAATTAA | |||||||| ||||||||||||||||||||||||||||||||||||||||||| CTGGGAGCCGCGAGGTCAATTGATTGAAACAACTTTGAACTTGTTGATCTTGTCACAAATTAA |||||||||||||||||||||||||||||||||||||||||||||||||||||||||| $\mid$

\section{vvl-53}

$\begin{array}{lll}\text { Cerat } 521195 & \text { TGATTGACGCGATCATGATTTACACCTT--TGGGTGCGTA } & 521232 \\ \text { Drase } 6839061 & \text { TGATTGACATGATCATGATTTACACCTTTCTGGGTGCTA } & \mathbf{6 8 3 9 1 0 0} \\ \text { Musca } 89266 & \text { TGATTGACATGATCGTGATTMACACCATCCCTCTGTTGTT } & 89291\end{array}$




\section{Supplemental Figure 2}

\begin{tabular}{|c|c|c|c|c|c|}
\hline \multicolumn{3}{|c|}{ Anopheles gambiae } & \multicolumn{3}{|c|}{ Anopheles gambiae-S1 } \\
\hline \multicolumn{3}{|c|}{ Composite eBLAT } & \multicolumn{3}{|c|}{ Composite eBLAT } \\
\hline Score & Start & End & Score & Start & End \\
\hline 1420 & 1 & 1420 & 1405 & 1 & 1420 \\
\hline 87 & 15 & 992 & 84 & 916 & 1172 \\
\hline 77 & 332 & 1061 & 80 & 376 & 1118 \\
\hline
\end{tabular}

\section{Anopheles christyi}

Composite eBLAT

Score Start End

$940 \quad 50 \quad 1390$

$\begin{array}{lll}75 & 50 \quad 168\end{array}$

$92 \quad 286 \quad 507$

C $1^{\text {st }} \bigcirc$ All Alignments

\section{Anopheles epiroticus}

Composite eBLAT

Score Start End

733

123

$84 \quad 329 \quad 716$

- $1^{\text {st }} \bigcirc$ All Alignments
Anopheles melas

Composite eBLAT

Score Start End

137411420

$\begin{array}{lll}73 & 322 & 517\end{array}$

$50 \quad 332 \quad 484$

- $1^{\text {st }} \bigcirc$ All Alignments

Anopheles culicifacies

Composite eBLAT

Score Start End

$535 \quad 137 \quad 1382$

$105 \quad 476 \quad 953$

$\begin{array}{lll}86 & 332 & 429\end{array}$

- $1^{\text {st }}$ All Alignments

Culex pipiens

Composite eBLAT

Score Start End

$204 \quad 44 \quad 1172$

$\begin{array}{lll}109 & 31 \quad 578\end{array}$

$85 \quad 44 \quad 922$

- $1^{\text {st }}$ All Alignments
Anopheles merus

Composite eBLAT

Score Start End

135711420

$\begin{array}{lll}76 & 332 & 1065\end{array}$

$87 \quad 341 \quad 965$

C $1^{\text {st }}$ All Alignments

Anopheles funestus

Composite eBLAT

Score Start End

$534 \quad 137 \quad 1384$

$131 \quad 285 \quad 974$

$\begin{array}{lll}99 & 33 & 356\end{array}$

- $1^{\text {st }} \bigcirc$ All Alignments

Aedes aegypti

Composite eBLAT

Score Start End

$181 \quad 245 \quad 824$

$172 \quad 245 \quad 824$

$71 \quad 761 \quad 1055$

$\begin{array}{lll}84 & 5 & 300\end{array}$

All Alignments

- $1^{\text {st }} \bigcirc$ All Alignments 
bioRxiv preprint doi: https://doi.org/10.1101/696005; this version posted July 11,2019 . The copyright holder for this preprint (which was not certified by peer review) is the author/funder, who has granted bioRxiv a license to display the preprint in perpetuity. It is made available under aCC-BY-NC-ND 4.0 International license.

\section{Supplemental Figure 3}

Apis_Mellifera (Bee) hth Genomic Relaxed EvoPrint

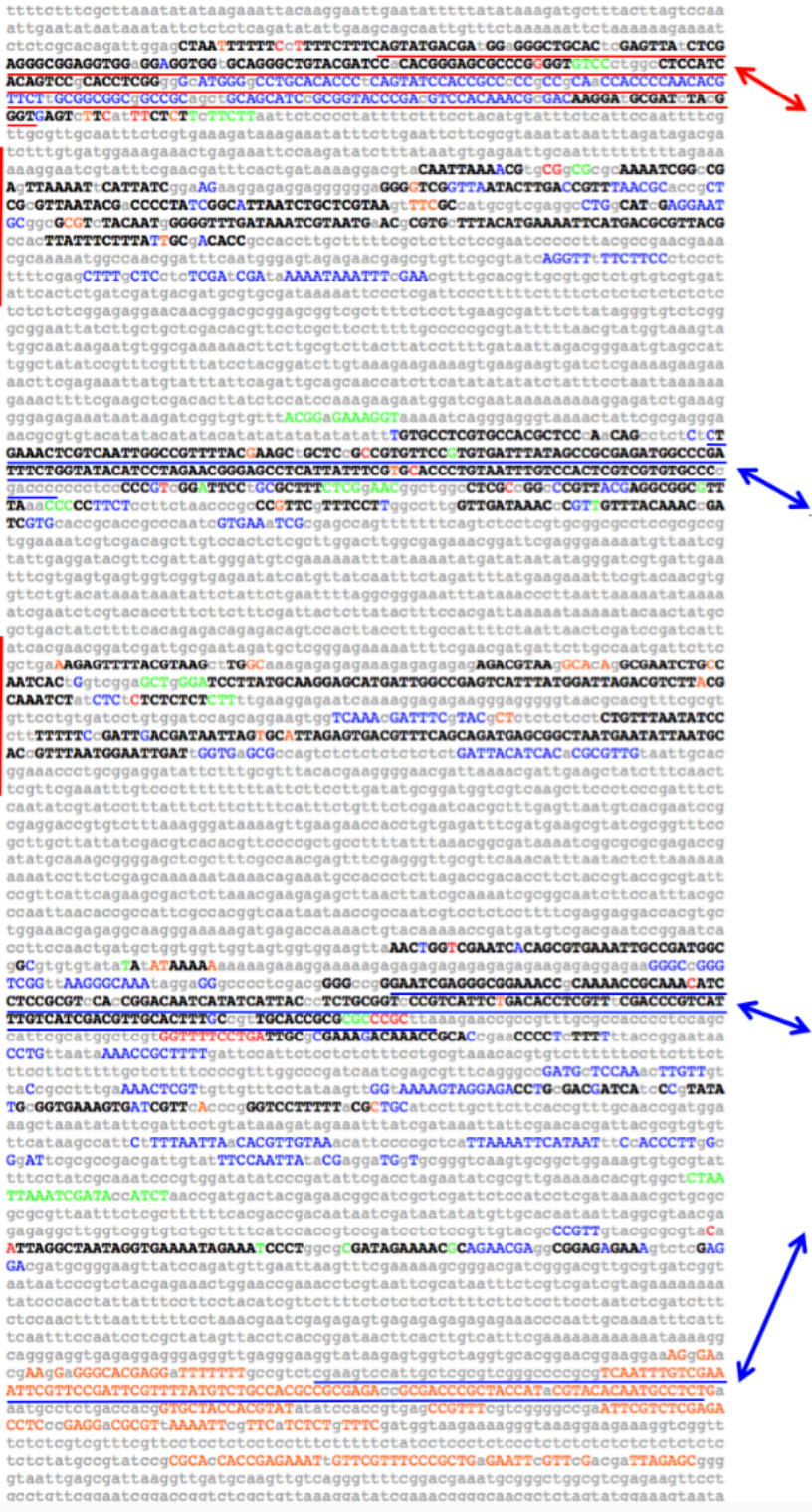

Cerapachys_Biroi(Ant) hth Genomic Relaxed EvoPrint

cagattagtcogatt tgctgatttgatatcogatactgattctaatcagaaacataggocgagcgaaatcgtatt

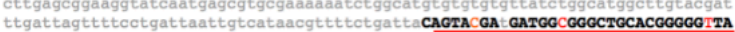

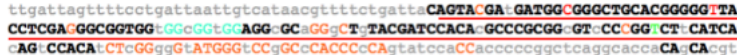

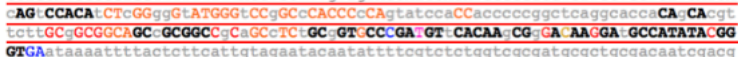
agacgagegtategegtgcgataaccgagcgeggt tgcgaacgttgcacgtatcaatagctctctcaaaccactat caaactot tgaatgcgacatatcacgaatttaagaattogatttacgaaaa tagataagtggaatctgcatata aatgtgtggacatgattacattgaaatatgogtataatcgtot togattatatagacgtatgtatcgttattgot

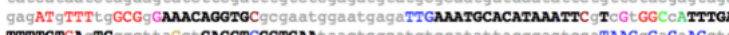
CCTCCCrCAn taactogcatgtocatattacggactocaTAAC

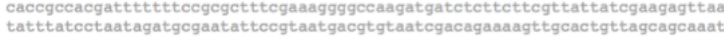

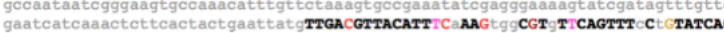
GCGCGATATCGTTTTTG GATACAT GCANAGTCG ACAGMTA CATTACCATA GGTTA CTC A AGATA

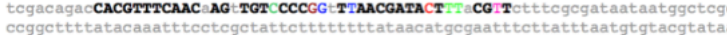
aaat tatcgttgaaagaagcact ttatattataatt tactettttcaaaaagctctcaacgtccatcaaat:

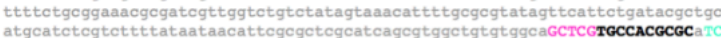
CGT ACgag CTGAAACACGTCAATTGGCCGTTT ACggAGCG GCTCgGCGGCC GTTCCGCGTGATTTATAG

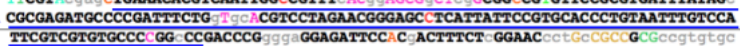
CaCgCT GAA ccagtogogcagoggtgtctaacttgggttactttgacgt taaagattagotogggtoggataccaattogettc cotgagagegagataaatatagaatgtggatacagtttgcattgcgtccototoggcgtttgatttogggaaag ctcatactattattatactacagcaatt tttoggtgcgotaaacaaaagagtttggatacgotaagaataaco

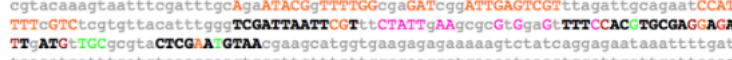

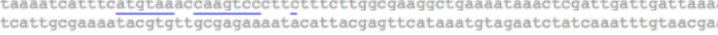
acatctttagcaacatatttettcacgatatatttctatatttttcaaacttatacogttgagttatcacagc tttcaqtcatgcttagaatgaagaatggeqtcataaacgtttcagacctgtgctgtaaaaaaagttacgeagte gcaqtegt gatgat ttt tctat tgtgacttaagtagtctcctctcctcteggtgtagttttacgtaacagccg

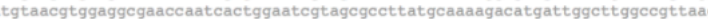
catctcacgaagcttttatgogaatccgaqtgacaatt tatcgccgacagacgcaatgatatctttcacttatcg .ttactoggtgaaacaattatgcagatagatogagtttccatgcggcaatttaactcgtataattaaact tt t

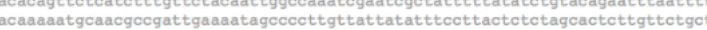
gcattttcottttcqgttcotctcaacatattcccotaactgctatttgactttatttgtgagtttattttggg cratactgatcgatacaataatatt th at tcgct

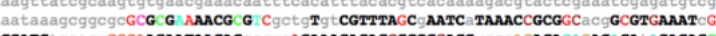

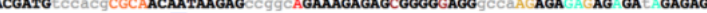
A I G GAGGCGTCGATT GAGGGGCGGAAACC CA AACCGCAAACATCCCCC $T$ TtaaCG CAACGSCCCAATC

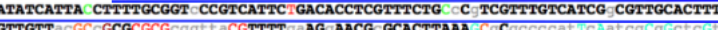
GTrG

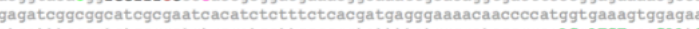

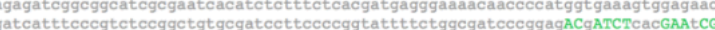

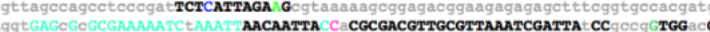

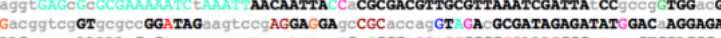

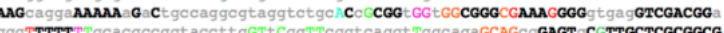

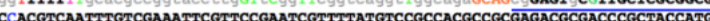

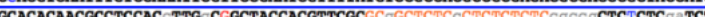

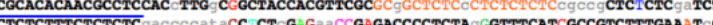

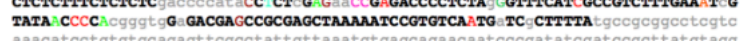
tgttgttagattogttgcactagtcttatgttttgaattctcteggtatttaattgtgactggaaaccatcag 


\section{Figure Legends}

Figure 1. Ultra-conserved sequences shared among a Drosophila ventral veins lacking enhancer and orthologous DNA within the Ceratitis capitata and Musca domestica genomes.

A) An EvoPrint of the D. melanogaster $v v l-41$ neuroblast enhancer showing 1,775 bp, located $26.6 \mathrm{~kb} \mathrm{3'}$ ' of the $v v l$ transcribed sequence. Capital letters represent bases in the $D$. melanogaster reference sequence that are conserved in D. simulans, D. sechellia, $D$. yakuba, D. erecta, D. ananassae, D. persimilis, D. grimshawi, D. mojavensis and D. virilis orthologous DNAs. Lower case grey bases that are not conserved in one or more of these species. Conserved sequence blocks (CSBs) shared with Ceratitis and Musca, as detected using BLASTn, DNA Block Aligner and the EvoPrinter CSB aligner are shown in Green text while red bases are shared between D. melanogaster and Ceratitis but not with Musca. B) Two and three-way alignments between of the ultra-conserved CSBs using BLASTn alignments. Green and red font annotations in the Drosophila CSBs are as describe above. Yellow highlighted bases in Ceratitis and Musca are not shared in Drosophila. Flanking BLASTn designator numbers indicate genomic sequence positions.

Figure 2. Ultra-conserved Drosophila, Ceratitis capitata and Musca domestica sequences within the homothorax locus.

A) A 1,065bp EvoPrint of the D. melanogaster homothorax locus that includes 5' nontranscribed sequence, its 5' UTR, the first five codons of its encoded protein and 102bp of the first intron. Capital letters represent bases in the $D$. melanogaster reference sequence that are conserved in D. simulans, D. sechellia, D. yakuba, D. erecta, D. ananassae, D. persimilis, D. grimshawi, D. mojavensis and D. virilis orthologous DNAs. Lower case grey letters represent bases that are not conserved in one or more than one of the test species. Drosophila CSBs that are shared with Ceratitis and Musca, as detected in figure 1, are shown in green. Red bases are shared only between Drosophila and Ceratitis and blue text represent bases shared exclusively between Drosophlia and Musca. The translation start codon is marked by an underlined ATG. B) BLASTn two and three-way alignments of the ultra-conserved CSBs. Font color annotations are as in panel A. Yellow 
highlighted bases in Ceratitis and Musca are not shared in Drosophila. Flanking BLASTn designator numbers indicate genome base positions.

Figure 3. EvoPrint analysis of the intragenic region adjacent to the Anopheles Wnt-4 and wingless genes identifies ultra-conserved sequences shared with the evolutionary distant Culex pipiens and Aedes aegypti genomes.

A) Anopheles gambiae genomic EvoPrint that spans 1,420 bp, located $10.2 \mathrm{~kb}$ upstream of the $W n t-4$ gene and $27.5 \mathrm{~kb}$ upstream of the wingless gene which is transcribed in the opposite orientation of Wnt-4 transcription. Capital letters (all font colors) represent bases conserved in all or all but one of the following Anopheles test species: A. gambiae-S1, A. merus, A. melas, A. epiroticus, A. christyi, A. funestus, A. culicifacies, A. dirus or A. farauti. Lower case grey letters represent bases that are not conserved in two or more of the Anopheles species included in the relaxed EvoPrint. Green uppercase bases indicate sequences are conserved in the Anopheles species, Culex pipiens and Aedes aegypti, blue font indicates Anopheles sequences that are shared only between Culex pipiens but not with Aedes aegypti and red font sequences are present only in Anopheles and Culex. B) To confirm the shared ultra-conserved CSBs, two and three-way BLASTn alignments of the shared sequences are shown. Color coding is as in panel A and yellow highlighted bases in the three-way alignments indicate identity between Culex and Aedes that is not present in Anopheles. Flanking BLASTn designator numbers indicate genome base positions.

\section{Figure 4. Conserved sequence clusters within the honeybee $d s c a m 2$ gene second intron.}

EvoPrinter analysis reveals Apis mellifera non-coding sequence elements that are conserved in other bee species or only in a subset of species. A) Alignment data generated from oneon-one comparisons of a $2.8 \mathrm{~kb}$ sequence from the honeybee $16 \mathrm{~kb}$ dscam 2 second intron. For each species, the top three independent $e$ BLAT alignment scores are listed. Scores indicate the total number of bases within the reference sequence, the Apis mellifera dscam 2 intron, that align with the test species genome. The test species; Apis dorsata, Apis florea, Bombus terrestrialis, Bombus impatiens, Habropoda laboriosa and Megachile rotundata are listed ( $\mathrm{L}->\mathrm{R}$ ) based on their highest alignment score in descending order. Website links to individual $e \mathrm{BLAT}$ alignments and superimposed composite $e$ BLATs are indicated in either red or blue font colors. As indicated in the alignment scorecard by the blue selection buttons, the top (highest scoring alignment) for each test species has been selected for EvoPrinting. 
B) A color-coded relaxed EvoPrint of the $2.8 \mathrm{~kb}$ honeybee $d s c a m 2$ second intron generated from the alignment data shown in panel A. Black uppercase letters indicate bases conserved in all test species. Font colors represent sequences conserved in all species except for Apis dorsata, Apis florea, Bombus terrestrialis, Bombus impatiens, Habropoda laboriosa or Megachile rotundata. Gray lowercase nucleotides are not conserved in at least two of the test species.

Figure 5. Combined Ant and Bee EvoPrints identify ultra-conserved Hymenoptera DNA

A) An Apis mellifera goosecoid (gsc) EvoPrint generated with four evolutionary divergent bee genomes and then overlaid with a print that includes the four bee genomes plus four divergent ant species. The Apis honeybee gsc DNA (1,701 bp) includes 5' non-coding, the first exon and intron sequences. All uppercase bases (both black and red font) are conserved in bees and sequences that are conserved in both bees and ants are denoted with red-font uppercase bases. Lowercase graycolored bases are not conserved in one or more of the bee test genomes. Bee test genomes: Bombus terrestrialis, Bombus impatiens, Habropoda laboriosa and Megachile rotundata. Ant test genomes: Linepithema humile, Monomorium pharaonis, Wasmannia auropunctata and Atta cephalotes. B) EvoPrints of the ant Wasmannia auropunctata castor (cas) gene locus. The 3,078 bp Wasmannia genomic DNA includes cas 5' non-coding, the first exon and flanking intron genomic sequences. The initial Evoprint was generated with four evolutionary divergent ants and then super-imposed with a print that included these four ants plus four bee genomes. All uppercase bases (both black and red font) are conserved in the ants Cerapachys biroi, Linepithema humile, Atta cephalotes and Vollenhovia emeryi. Sequences that is conserved in both ants and bees (Apis florea, Bombus impatiens, Habropoda laboriosa and Megachile rotundata) are shown as red colored uppercase bases. Lowercase gray-colored bases are not conserved in one or more of the ant test species. The translation initiation codon is underlined. The left flanking vertical brown bar indicates an ant-specific conserved DNA cluster that is not found in bees. Note, in the exon ORF most, but not all, of the conserved codons do not have conserved wobble positions indicating that the cumulative evolutionary divergence of the test species used to generate the EvoPrint afford near base pair resolution of essential DNA. 
Figure 6. Side-by-Side comparison of conserved sequences within in the bee and ant glass bottom boat loci identify clusters of conserved and species-specific sequences.

A) Relaxed EvoPrint of Apis mellifera genomic DNA that includes the glass bottom boat $(\mathrm{gbb})$ second and third exons (red underlined sequences) plus flanking intronic sequences $(6.6 \mathrm{~kb})$. Black uppercase bases are conserved in all test bee species and colored uppercase bases are conserved in all but one of the color-coded test species: Bombus terrestrialis, Habropoda laboriosa, Megachile rotundata and Bombus impatiens. First and second exons sequences underlined red. Blue underlined sequences are homologous to underlined sequences in panel B. Vertical red bars flanking the EvoPrint indicate conserved bee-specific sequences that are not found in ants. B) Relaxed EvoPrint of Wasmannia auropunctata DNA that spans the second and third exons of the $g b b$ gene including their flanking intronic sequences $(5.1 \mathrm{~kb})$. As in panel A, black uppercase bases are conserved in all test ant species and colored uppercase bases are conserved in all but one of the color-coded species: Cardiocondyla obscurior, Cerapachys biroi and Linepithema humile. Red and blue underlined sequences are respectively homologous coding and non-coding sequences in panel A and the green vertical bar flanking the EvoPrint indicates ant-specific conserved sequences that are not found in bees.

\section{Supplemental Figure 1. Ultra-conserved DNA in Drosophila vvl enhancers identified in Ceratitis capitata and Musca domestica orthologous DNAs.}

Three-way Ceratitis-Drosophila-Musca BLASTn alignments of CSBs within six different in vivo tested Drosophila $v v l$ enhancers. Drosophila sequences that are shared with Ceratitis and Musca are shown in green. Red bases are shared only between Drosophila and Ceratitis and blue text represent bases shared exclusively between Drosophila and Musca. Yellow highlighted Ceratitis and Musca bases are not shared in Drosophila. Flanking BLASTn designator numbers indicate genomic base positions.

\section{Supplemental Figure 2. Conservation within the mosquito wingless gene second intron.}

EvoPrinter analysis reveals Anopheles gambiae non-coding sequence elements located between the mosquito homologs of Drosophila $w g$ and wht4 that are conserved in other mosquito species. Alignment data generated from one-on-one comparisons of a 1420 base sequence from the $A$. gambiae genome. For each species, the top three independent $e$ BLAT 
alignment scores are listed. Scores indicate the total number of bases within the reference sequence that align with the test species genome. In this analysis, 11 of the 19 mosquito test species present in the database are illustrated. The test species are listed $(\mathrm{L}->\mathrm{R})$ based on their highest alignment score in descending order. Website links to individual $e \mathrm{BLAT}$ alignments and superimposed composite $e$ BLATs are indicated in either red or blue font colors. As indicated in the alignment scorecard by the blue selection buttons, the top (highest scoring alignment) for each test species has been selected for EvoPrinting.

\section{Supplemental Figure 3. Side-by-side comparison of conserved sequences within ant and bee homothorax loci identifies shared exon/intron architecture and species- specific conserved sequences.}

EvoPrints of bee and ant genomic DNA that includes homothorax (hth) encoding an exon isologous to the $2^{\text {nd }}$ exon of Drosophila hth plus flanking intronic sequences. Blue and red underlined regions are coding and non-coding sequences, respectively, and align with homologous regions in the two panels. Black uppercase bases are conserved in all test species and colored uppercase bases are conserved in all but one of four bee tests species in panel A and all but one of three ant test species in panel B. A) Relaxed EvoPrint of Apis mellifera genomic sequences $(6.3 \mathrm{~kb}$; Group5:7,111,526-7,117,900). Vertical red bars flanking the EvoPrint indicate conserved bee-specific sequences that are not found in ants. Colored uppercase bases are conserved in all but one of the color-coded test species: Apis florea, Habropoda laboriosa, Bombus terrestrialis and Bombus impatiens. B) Relaxed EvoPrint of Cerapachys biroi genomic DNA (5.1kb; 6532628-6527517, Ooceraea biroi isolate clonal line C1 chromosome 14, Obir_v5.4). The green vertical bar flanking the EvoPrint indicates ant-specific conserved sequence that in absent in bees. Black uppercase bases are conserved in all test ant species and colored uppercase bases are conserved in all but one of the color-coded test species: Monomorium pharaonis, Atta cephalotes, 